\title{
Floristic Study for Tarhuna-Libya
}

\section{Tarhuna-Libyanın Floristik Çalışması}

\author{
Research Article
}

Mohammed Hadi Mahklouf*', Abdurrazag S. Sherif², Abdalla G. Betelmal²

'Botany Department, Faculty of Sciences, Tripoli University, Tripoli,Libya.

${ }^{2}$ Natural Reassures Department, Faculty of Agriculture, Tripoli University, Tripoli, Libya.

\section{A B S T R A C T}

survey of plant species of Tarhuna was taken in between 2016-2017. A total number of 518 plant specimens have been collected from Tarhuna representing 41 families, 143 genera, and 222 different plant species of which 36 species are belonging to monocotyledons, 185 species belonging to dicotyledons, and 1 species belong to Gymnosperms Ephedera alltisma. A total of 126 plant species were reported for the first time from Tarhuna. The results of this study have shown that the dominance of the family Asteraceae with 42 species, followed by the family Fabaceae with 30 species, the family Poaceae with 27 species, and the family Lamiaceae with 11 species. Anthemis glarosa endemic species for Libya confined to the costal belt of Libya has been collected, identified, and reported for the first time from Tarhuna.

\section{Key Words}

Flora Libya, Tarhuna, diversity, flowering plants.

\section{öz}

016-2017 yılları arasında Tarhuna bitki türlerinin bir araştırması yapılmıştır. Tarhuna'dan 41 familya, 143 cins

ve 36 türün monokotiledonlara ait olduğu 222 farklı bitki türü, dikotiledonlara ait 185 tür ve Gymnosperms Ephedera alltisma'ya ait 1 türden toplam 518 bitki örneği toplanmıştır. Tarhuna'dan ilk kez toplam 126 bitki türü bildirildi. Bu çalışmanın sonuçları, Asteraceae familyasının 42 türle baskın olduğunu, bunu 30 türle Fabaceae ailesinin, 27 türle Poaceae ailesinin ve 11 türle Lamiaceae ailesinin izlediğini göstermiştir. Libya'nın kıyı şeridiyle sınırlı olan Libya'ya yönelik Anthemis glarosa endemik türleri toplanmış, tanımlanmış ve Tarhuna'dan ilk kez rapor edilmiştir.

\section{Anahtar Kelimeler}

Libya florası, Tarhuna, çeşitlilik, çiçekli bitkiler .

Article History: Received: Feb 5, 2018; Revised: Mar 3, 2018; Accepted: Sep 3, 2018; Available Online: Oct 4, 2018

DOI: $10.15671 /$ HJBC.2018.242

Correspondence to: M.H. Mahklouf, Botany Department, Faculty of Sciences, Tripoli University, Tripoli,Libya. 


\section{INTRODUCTION}

This his paper deals mainly with the flora of Tarhuna which is located about $88 \mathrm{~km}$., south east of Tripoli (Capital) and occupies an area approximately $3820 \mathrm{~km}^{2}$ between $32^{\circ} 26^{\prime} \mathrm{N}$ latitude $\& 13^{\circ} 38^{\prime} \mathrm{E}$ longitude, and it is about 350 Tarhuna is bounded by Gharabulli to the north, Msallata \& Zliten to the east, Gharian \& BenGhisher to the west and Beni-Walid to the south (Figures 1, 2, 3). The climate of Tarhuna followes the climate of the Mediterranean region which is cold \& rainy at the winter with an averge rainfall ranges between $100-300 \mathrm{~mm}$ annually, and hot $\&$ dry at the summer with a mean of $18^{\circ} \mathrm{C}$ [1]. In general the scarcity of rainfall make the region arid- semiarid, such condition makes the flora of Tarhuna poor with regard to its vast area. The history of plant exploration in Libya has been done by several authors such as (2-5, 9-16). Since the flora of Tarhuna has not been studied thoroughly during the work on the flora of Libya (1975-1989). Therefore, the purpose of this survey is to have an exclusive study to its flora.

\section{METHODS} $500 \mathrm{~m}$ above the see level as measured by GPS

A total number of 518 plant specimens have been collected between 2016-2017 upon various field trips about one trip per month. The collected plants were then treated by the usual herbarium procedures including pressing, poisoning, mounting, labeling, and identifying. Identification of plant species was done by using the Flora of Libya $[1,6-8]$. Eventually, the identified plant specimens were deposited at the national herbarium, Botany Department, Faculty of Sciences, Tripoli University. Collection of plant specimens was done by the authores, Mahklouf, Sherif, and Betelmal [MSB].

\section{Enumeration of the Plant Species}

This enumeration includes 222 identified plant species of which 126 species were reported for the first time from Tarhuns. The new reported species are marked with an asterisk. Families and species included in this enumeration are arranged alphabetically.

\section{Alliaceae}

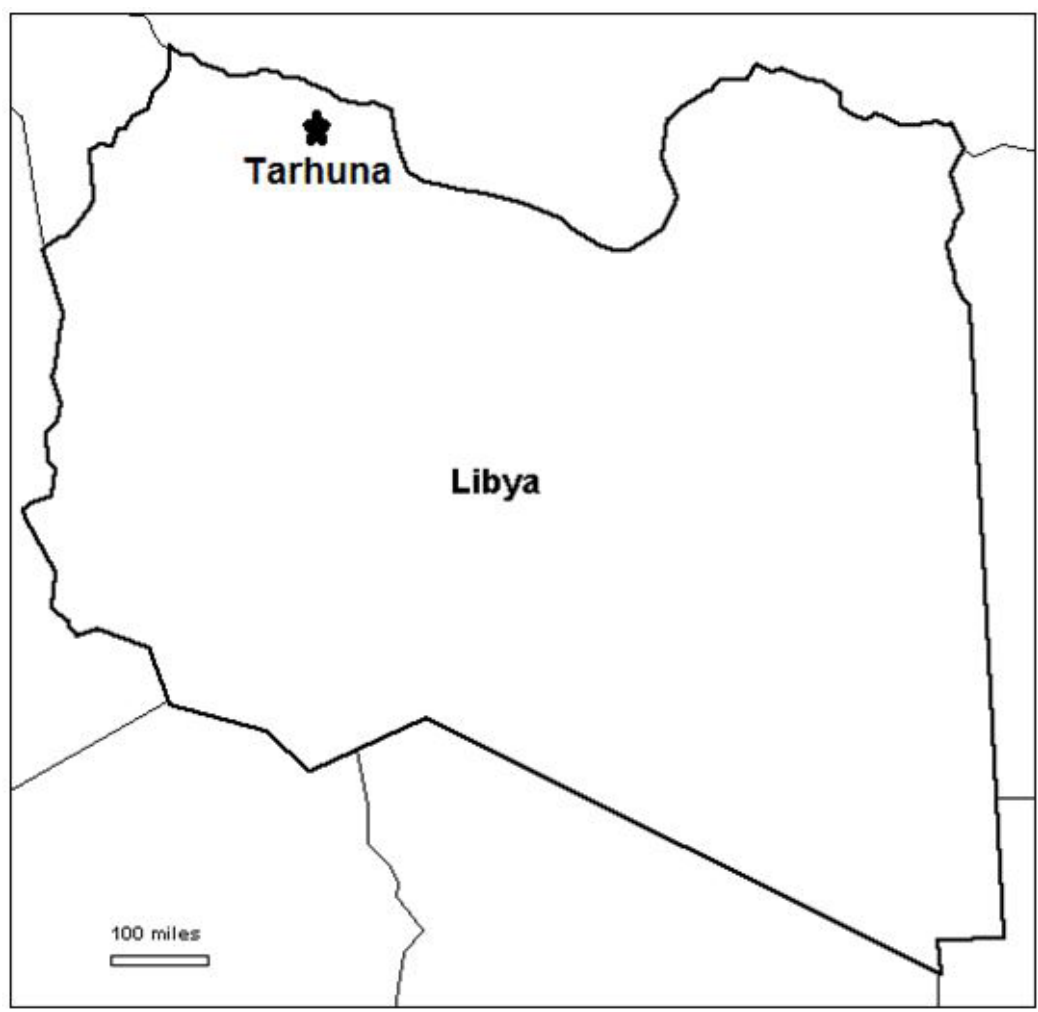

Figure 1. Map o Libya showing Location of Tarhuna. 


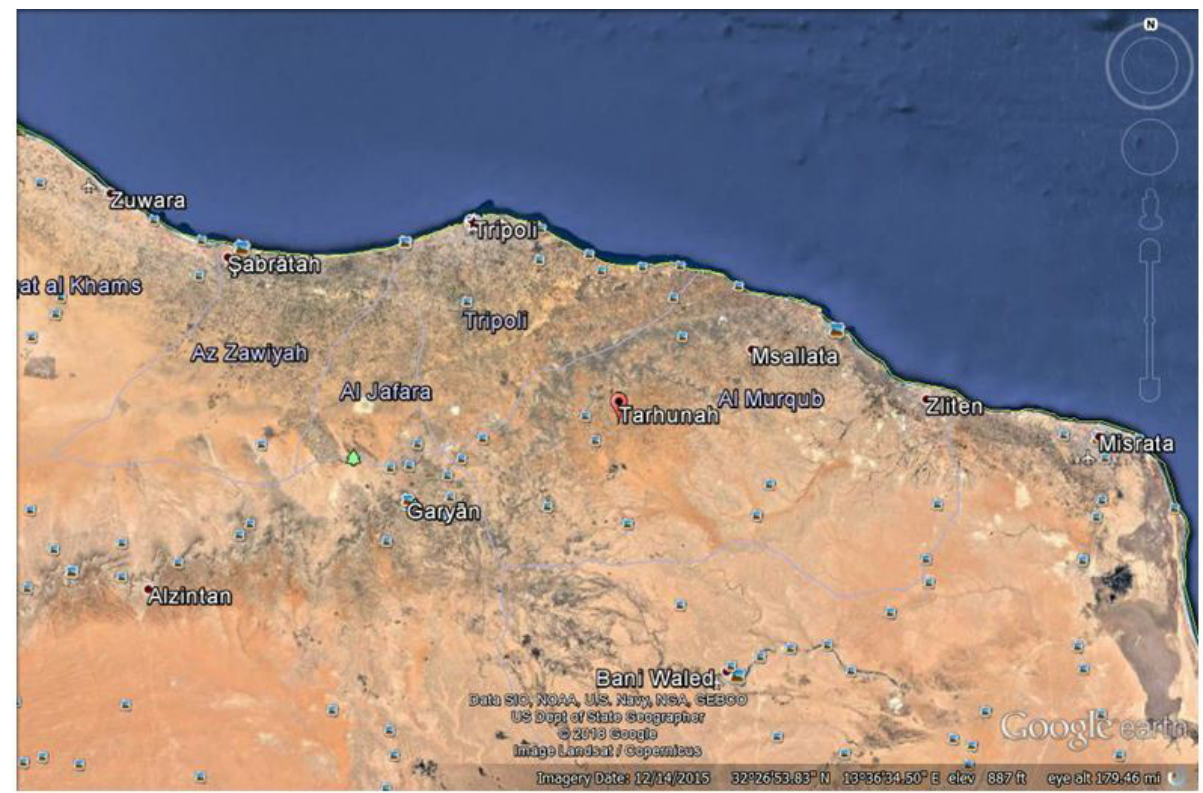

Figure 2. Map pf Nort-West part of Libya showing Location of Tahuna.

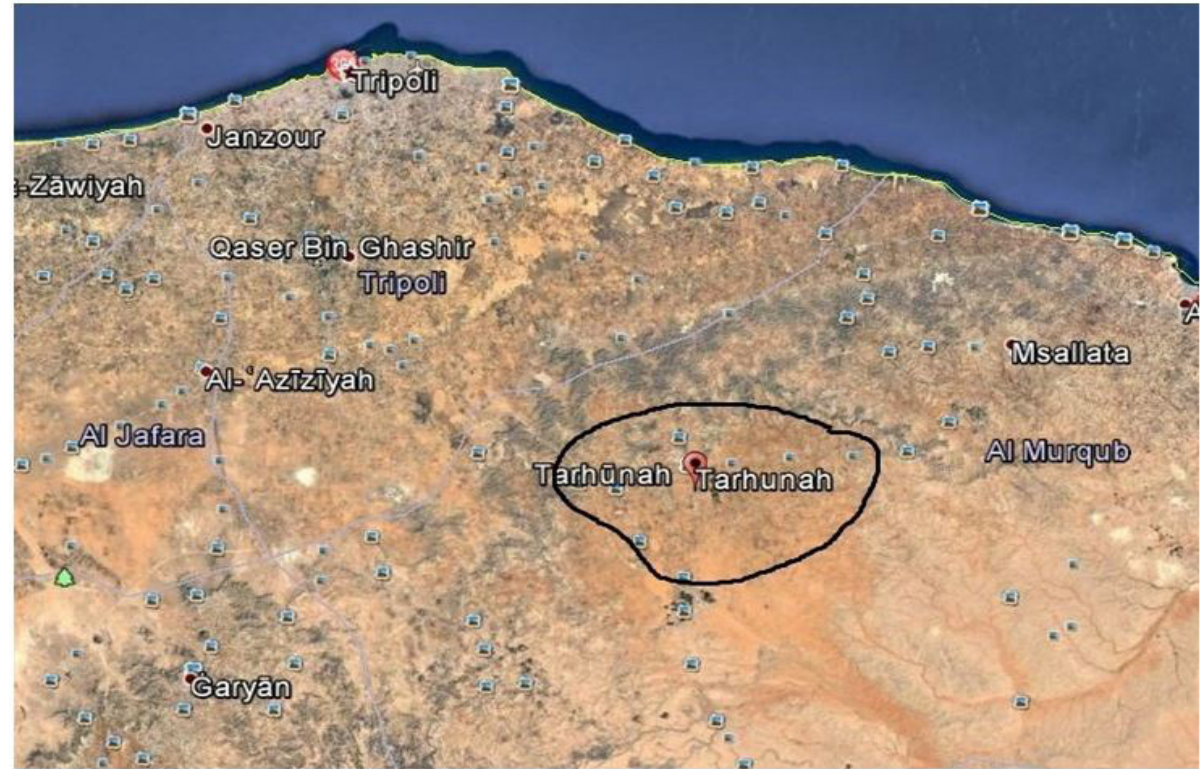

Figure 3. Map of Tarhuna showing Locaion of stuy area.

1.1. Allium roseum L., Sp. Pl.296. 1753.

Collection number: 828

Distribution: East Mediterranean element.- In Tarhuna: Msabha.

Fl: Mar. - April

Collectors: MSB Collection date: 19.05.2016

\section{Anacardiaceae}

2.1. *Pistacia atlantica Desf., PI. Atl. 2: 364.1799. Collection number: 904

Synonyms: Pistacia mutica Fisch. \& Mey., in Hoen., Enum. PI. Talysch 102. 1837.

Distribution: Canary Islands, Mediterranean region, N. Africa, Anatolia, Cyprus, N. E. Greece, Crimea, Caucasia, Iran, Afghanistan and Pakistan. - In Tarhuna: Terghlat.

FI: March-April

Collectors: MSB Collection date: 10.05.2017 2.2.*Rhus tripartita (Ucria) Grade in Bull. Orto Bot. Napoli 5: 62.1918.

Collection number: 829

Synonyms: Rhamnus tripartita Ucria in Roem., Arch. i. 1: 68. 1796; Rhus oxyacantha auct., non Schousboe ex Cav. Icon. 111, 36. 1794: Durand \& Barratte, I.c. 61.

Distribution: Dry places in Sicily, Malta, N. Africa, 
S. W. Asia. - In Taruna: Msabha.

FI. October-June.

Collectors: MSB Collection date: 19.05.2016

\section{Apiaceae}

3.1. Bubpeurum semicopositum L., Demonst. PI. Hort. Upsal. 7. 1753.

Collection number: 808

Synonyms: Bubpeurum glaucum Robill. et Cast. In Lam. \& DC., Fl. Franc. 5: 515. 1815. B.semicopositum var. glaucum (Robill. et Cast.) Paol. In Fiori et Paol., Fl. Anal. Ital. 2: 155. 1900.

Distribution: S. Euorpe, N. Africa, Mediterranean Islands, Balkans, Turkey, Palestine, Russia and S. W. Iran. - In Tarhuna: Arabeen and Wlaad Hammad.

FI.March-May.

Collectors: MSB Collection date: 10. 05.2017

3.2.*Daucus sahariensis Murb., Contr. Fl. Tunisie etc. 1: 88, pl. 5. 1897.

Collection number: 853

Synonyms: Platyspermum pubescens Pomel, Nouv. FI. Atl. 148. 1874, non Daucua pubescens Koch,1824.

Distribution: N. Africa (Algeria, Tunnis, Libya, Egypt). - In Tarhuna: Dawoon.

FI.April-June.

Collectors: MSB Collection date: 27.04.2017

3.3. Daucus syrticus Murb., Contr. Fl. NordOuest Afr. 1: 87. 1897.

Collection number: 900

Distribution: N. Africa. - In Tarhuna: Arabeen.

FI. March-April.

Collectors: MSB Collection date: 10.05.2017

3.4.*Ferula tingitana L., Sp. PI. 247. 1753.

Collection number: 840

Distribution: S.W. Euorpe, N.W. Africa, Libya, Palestine \& W. Syria. - In Tarhuna: Khsaya.

FI. February-March.

Collectors: MSB Collection date: 27.04.2017

3.5. Pithoranthus tortuousus (Desf.) Bentham \&Hoker, Gen. Plant. 1 (3): 890. 1867

Collection number: 854

Synonyms: Bubon tortuosum Desf., Fl. Atlant. 1: 360. 1798; Deverra tortuosa (Desf.) DC., Prodr. 4: 143. 1830 .

Distribution: N. Africa, Palestine. - In Tarhuna:
Dawoon and Terghlat.

FI: Throughout the year.

Collectors: MSB Collection date: 27.04.2017

3.6.*Thapsia garganica L., Mantissa 57. 1767.

Collection number: 830

Synonyms: Thapsia decussata Lag., Gen. et Sp. Nov. 12. 1816; T. praealta d'Urv., Enum. PI. Ins. Pont. Eux. 288. 1822; T. sylphium Viv., Fl. Lib. Spec. 17. 1824; T. garganica var. sylphium (Viv.) Aschers.

Distribution: N. Africa (Libya). S. \& S. W. Europe and Turkey. - In Tarhuna: Msabha.

FI. April-May.

Collectors: MSB Collection date: 19.05.2016

\section{Asclepiadaceae}

4.1.*Calotropis procera (Ait.) Ait. F., Hort. Kew. Ed. 2.2: 78:.1811.

Collection number: 905

Synonyms: Asclepias procera Ait., Hort. Kew. 1: 305. 1789.

Distribution: Libya, Tropical to dry sand parts of Africa, Palestine, Abu Dhabi, W. India, Brazil, CoIombia and Venezuela. - In Tarhuna: Terghlat.

FI. Throughout the year.

Collectors: MSB Collection date: 12.06.2017

\section{Asteraceae}

5.1.*Anacyclus monanthos (L.) Thell. In Mem. Soc. Nat. Hist. Cherbourg ser. 4,38:518.1911.

\section{Collection number: 693}

Synonyms: Tanacetum monanthosL., Mantissa 111. 1767; Santolina terrestris Forsk. Fl. Aegypt. 147. 1775; Anacyclusalexandrinus Willd. , Sp.PI. ed. 4. 3: 2173.1803; Anacyclus alexandrines f. capillifolius Pamp., Bull. Soc. Bot. Ital. 1914: 19: 1914.

Distribution: N. Africa to Palestine. - In Tarhna: Dawoon, Meghawsha, Sok El-Ahed, Terghlat and Whedat.

FI.February-May

Collectors: MSB Collection date: 19.03.2016

5.2. Anthemis glareosa Durand \& Barratte, Fl. Lib. Prodr. 130. 1910.

Collection number: 780

Distribution: Endemic to N. coastal belt of Libya. - In Tarhuna: Zurgan.

FI. January-April.

Collectors: MSB Collection date:8.04.2016 5.3. Anthemis secundiramea Biv., Sic. PI. Cent. 2:10. 1806. 
Collection number: 750

Synonyms:Anthemis indurateDelile, Descr. Egypte, Hist. Nat. 2:274t. 43.1813; Pamp., PI. Trip. 245. 1914; Tackh., St. Fl. Egypt ed. 2, 574. 1974; A. secundiramea var. indurate (Delile) DC. Prodr. 6: 10. 1838; A. indurate var. angulata Pamp., in Bull. Soc. Bot. Ital. 1914; Keith, I.c.

Distribution: Mediterranean region. - In Tarhuna: Arabeen, Dawoon, Hejaz, Msaaed, andTerghlat.

FI.March-April.

Collectors: MSB Collection date:22.03.2016

5.4. *Artimisia campestris L., Sp. PI. 846, 1753. Collection number: 781

Synonyms: Artemisia glutinosa Gay ex Besser Tent. Abrot. 34. 1832; Pamp.,I.c.; Keith, I.c.; A. variabilisa Ten., Fl. Neap. Prodr. App. 5:28. 1835-38; Keith, I.c. 257.,A. campestris ssp. glutinosa (Gay ex Besser) Batt. \& Trabut. Fl. Alger. Dicot. 469. 1889; Tutin et al., 186. 1976.

Distribution: Europe, N. Africa. - In Tarhun: Dawoon, Msabha, Sok El-Ahed and Terghlat.

FI.November-January.

Collectors: MSB Collection date: 8.04 .2016

5.5.*Artimisia herba-alba Asso, Syn. Stirp. Arag. 177. 1779.

Collection number: 906

Synonyms: Artemisia arragonensis Lam.,Encycl. Meth. Bot. 2:269. 1783; A. inculta Delile, Descr. Egypte, Hist. Nat. 264. 1813; Tach., I.c.581; A. pyromacha Viv., Fl. Lib. Spc. 54. T. 13. 1824; A. inculta var. laxiflora (Boiss.) Tackh., I.c.

Distribution: W. Southern France, Spain. N. Africa, Syri - W. Iran. - In Tarhuna: Terghlat.

FI.November-February.

Collectors: MSB Collection date: 12.06.2017

5.6.*Atractylis cancellata L., Sp. PI.830. 1753. Collection number: 751

Synonyms: Atractylis caespitosa auct. Non Desf.: Viv., Fl. Lib. Spec. 52. 1824.

Distribution: Mediterranean area, up to N. Iraq and W. Iran. - In Tarhuna: Arabeen, and Hejaz.

FI. April- May.

Collectors: MSB Collection date:22.03.2016

5.7. Atractylis delicatula Batt. Ex Chevall. In Bull. Herb. Boiss. Ser. 2.3: 773. 1903.

Collection number: 881

Distribution: N. Africa (Southern Morocco, N. Al- geria and W. Libya). - In Tarhuna: Wlaad Hammad. FI.April-May.

Collectors: MSB Collection date: 10.05.2017

5.8.*Atractylis serratuloides Sieb. ex Cass., Dict. Sci. Nat. 50:56. 1827.

Collection number: 694

Synonyms: Atractylis microcephala Cosson \& Durieu in Ann. Sci. Nat. Ser. 4.1:240. 1854.

Distribution: N. Africa. - In Tarhuna: Hejaz, Meghawsha and Terghlat.

FI.May-June.

Collectors: MSB

Collection date:19.03.2016

5.9.Calendula arvensis L., Sp. PI. ed. 2, 1303. 1763.

\section{Collection number: 855}

Synonyms: Calendula sanctaL., I.C.; C. aegyptiaca Desf., Tabl. Ecole Bot. ed. 1, 100. 1804; C. cristagalli Viv.,Fl. Lib. Spec. 59t. 26. 1824; C. malvaecarpa Pomel, Nouv. Mat. Fl. Atl. 33:1874; C.aegyptiaca var. suberostris Boiss., Fl. Or. 419. 1875.

Distribution: A Mediterraneanelement, S. Europe, N. Afric- Caucasia, Iran and Afghanistan. - In Tarhuna: Dawoon, Sok El-Ahed and Terghlat.

Fl.November-April.

Collectors: MSB Collection date:27.04.2017

5.10.*Carduncellus eriocephalus Boiss., Diagn. PI. Nov. ser. 1, 10:100. 1849.

Collection number: 782

Distribution: N. Africa and Palestine. - In Tarhuna: Terghlat, Wlaad Hammad and Zurgan.

FI.March-June.

Collectors: MSB Collection date: 8.04.2016

5.11. *Carduncellus pinnatus (Desf.)DC., Prodr. 6:614. 1838.

Collection number: 842

Synonyms: Carthamus pinnatus Desf., Fl. Atl. 2:258 t. 229. 1799.

Distribution: N. Africa and S. Europe (Central Spain, Sicily). - In Tarhuna: Dawoon, Sok -El-Ahed and Whedat.

FI: March-April.

Collectors: MSB

Collection date:27.04.2017

5.12.*Carduus argentatus L., Mantissa Alt. 280. 1771.

Collection number: 735 
Distribution: East Mediterranean element; Libya, Egypt, Palestine, Syria, Crete, Cyperus, Greece, S. Anatolia and Iraq. - In Tarhuna: Arabeen, Hejaz, Msaaed, Sok El- Ahed, Terghlat, Whedat, and Zurgan.

FI. March-April.

Collectors: MSB

Collection date: 22.03 .2016

5.13.* Carduus getulus Pomel, Nouv. Mat. Fl. Atl. 2:275. 1875 .

\section{Collection number: 857}

Synonyms: Caduus pycnocephalus acut. non L.; Ascherson in Rohlf's Kufra 528. 1881.

Distribution: N. Africa. - In Tarhuna: Dawoon and wlaad Hammad.

FI.February-May.

Collectors: MSB

Collection date:27.04.2016

5.14. *Carthamus lanatus L., Sp. PI. 830. 1753. Collection number: 813

Synonyms: Carthamus creticusL., I.c. ed. 2, 1163. C. Ianatus var. longifolius Pamp. in Novu. Giorn. Bot. Ital. N.S. 31: 229. 1924.

Distribution: W. Central and S. Europe, and N. Africa. - In Tarhuna: Dawoon and Msabha.

FI. June-November.

Collectors: MSB

Collection date: 19.05 .2016

5.15. Centaurea dimorpha Viv., FI. Lib. Spec. 58 T. 24.1824.

Collection number: 783

Synonyms: Centaurea sonchifolia L. var dimorpha (Viv) DC.,Prodr. 6:599. 1839.

Distribution: N. Africa. - In Tarhuna: Arabeen, Dawoon, Msaaed, Terghlat and Zurgan.

FI.March-June.

Collectors: MSB Collection date: 8.04.2016

5.16. *Centaurea sphaerocephala L., Sp. PI. 916. 1753.

Collection number: 810

Synonyms: Centaurea polycantha Willd., Sp. PI. ed. 4, 3:2312, 1803; C. fontanesii Spach ex Durieu in Duchartre, Rev. Bot. 2:429.1847.

Distribution: A maritime species ofW.Mediterraneanregion. -In Tarhuna:Msaaed and Msabha.

FI. May.

Collectors: MSB Collection date: 19.05.2016

5.17. * Chyrsanthemun coronarium L., Sp. PI. 890. 1753.
Collection number: 736

Synonyms: Matricaria coronaria (L.) Desr. In Lam., Encycl. Meth. Bot. 3: 737.1792; Chrysanthemum umbrosum Willd. , Sp. Pl. ed. 4, 3:2149. 1803;Pinardia coronaria (L.) Less., Syn. Gen. Comp. 255. 1832

Distribution: Mediterranean region to N.W. Iran. InTarhuna: Sok El-Ahed, Terghlat and Whedat.

FI.March-June.

Collectors: MSB Collection date: 22.03.2016

5.18. *Crepis vesicaria L., Sp. PI. 805. 1753.

Collection number: 856

Synonyms: Crepis taraxacifolia Thuill., FI. Paris 409. 1799; C. scariosa Willd., Sp. Pl. ed. 4. 3: 1595. 1803; C. marschallii (C. A. Meyer) Schultz Bip. In Flora 23:718. 1840.

Distribution: Mediterranean region extanding into east in Crimea, Cauasia and N. up to Netherland and W. Austria- In Tarhuna: Dawoon and Sok El-Ahed.

FI.March-May.

Collectors: MSB

Collection date: 27.04 .2017

5.19. Cynara cardunculus L., Sp. PI. 827.!753. Collection number: 908

Distribution: Mediterranean region. - In Tarhna: Terghlat.

FI.April-May.

Collectors: MSB Collection date: 12.06 .2017

5.20. *Echinops galalensis Schweinf. In Ascherson \& Schwein. , Suppl. III. Fl. Egypte 2:762. 1889.

Collection number: 811

Distribution: N. Africa. - In Tarhuna: Msabha.

FI. May-June.

Collectors: MSB Collection date:19.05.2016

5.21. *Echinops spinosissimus Turra, Farset, Nov. Gen. 13. 1765.

Collection number: 882

Synonyms: Echinops bovei Boiss., Diagn. PI. Or. Nov. ser. 16: 19. 1845; E. spinosus acut. Non L. 1767. Distribution: Mediterranean region (S. Europe \& N. Africa).- In Tarhuna: Wlaad Hammad.

FI.March-May.

Collectors: MSB Collection date: 10.05 .2017

5.22.*Filago desertorum Pomel, Nouv. Mat. Fl. Atl. 46.1874.

Collection number: 841 
Synonyms: Filago spathulata acut. Non C. Presl. 1822.

Distribution: S. Spain, N. Africa and S.W. Asia. - In Tarhuna: Arabeen, Dawoon and Wlaad Hammad. FI. March-April.

Collectors: MSB Collection date:27.04.2017

5.23. *Filago mareotica Delile, Descr. Egypt, Hist. Nat. 2:274. 1813.

Collection number: 909

Synonyms:Gifolaria mareotica (Delile)Chrtek \& Holub, Preslia 35: 10. 1963.

Distribution: Africa and S. E. Spain. - In Tarhna: Terghlat.

FI.March-April.

Collectors: MSB

Collection date:12.06.2017

5.24. Filago pyramidata L., Sp. PI. 1199. 1753.

Collection number: 864

Synonyms: Filago germanica L. var. spathulata(C. Presl) DC., Prodr. 6:247.1838; F. jussiaei Cosson \& Germ. In Amnn. Sci. Nat. 20 (2): 284. 1846.

Distribution: S. Euorpe and N. Africa. - In Tarhuna: Sharshara.

FI. March-May.

Collectors: MSB

Collection date:27.04.2017

5.25. Filago ramosissima Lange Ind. Sem. Horto Haun. 1855.24.1855; Holub in Tutinet al. , I c. 122.

Collection number: 907

Synonyms: Filago mareotica auct. Non Delile (1913); Durand \& Barratte, I.c. 127.

Distribution: S. Spain and N. W. Africa. - In Tarhuna: Terghlat.

FI. March.

Collectors: MSB

Collection date: 12.06 .2017

5.26. *Hedypnois cretica (L.) Dum.Courset. Bot. Cult. 2:339. 1802.

\section{Collection number: 784}

Synonyms: Hyoseris cretica L., Sp. PI. 810. 1753; H. hedypnoisL., I.c. 809; Hedypnois globuliferaLam., Fl. Fr. 2: 107. 1778; Pamp. Prodr. FI. Cir. 470. 1931.

Distribution: Mediterranean region up to W. Iran.

- In Tarhuna: Zurgan.

FI. September-March.

Collectors: MSB Collection date: 8.04.2016

5.27.*Helichrysum lacteum Cosso \& Durieu in Bull. Soc. Bot. Fr. 2:365.1855.
Collection number: 752

Distribution: Morocco, Algeria and Libya. - In Tarhuna: Hejaz.

Fl. March.

Collectors: MSB Collection date:22.03.2016

5.28.*Helichrysum stoechas (L.) Moench, Meth. 575. 1794.

Collection number: 804

Synonyms: Gnaphalium stoechasL., Sp. PI. 853. 1753; Helichrysum stoechas (L.) Moench ssp. conglobatum (Viv.) Maire \& Weiller.

Distribution: Mediterranean Europe, N. Africa and Anatolia. - In Tarhuna: Msaaed and Msabha.

FI.April-May.

Collectors: MSB Collection date:19.05.2016

5.29. Hypochoeris glabra L., Sp. PI. 811. 1753. Collection number: 722

Synonyms: Hypochoeris minimaCyr., PI. Rar. Neap. 1:10. 1788; $\boldsymbol{H}$. arachnoidea Desf. Ex Poiret in Lam., Encycl. Meth. Bot. 5:572. 1804.

Distribution:. Europe, N. Africa. - In Tarhuna: Hejaz, Mckhalef and Zurgan

FI.March-April.

Collectors:MSBCollection date:19.03.2016

5.30.* Launaea capitata (Sprengel) DandyinF. W. Andrews, FI. PI. Sudan 3:40. 1956.

Collection number: 910

Synonyms:Sonchus capitatus Sprengel, Syst. Veg. 3:650. 1826; Launaea glomerata (Cass.) Hooker fil., Brit. India 3:416. 1881; Launaea glomerata f. minima Pamp. in Forti, Marc. e Pamp., Atti R. 1st Veneto Sc. Lett et Art; 92 (2): 236. 1932.

Distribution: Sahara-Sind element. - In Taruna: Whedat.

FI.February-June.

Collectors: MSB Collection date: 12.06.2017

5.31. Launaea resedifolia (L.) Kuntze, Revis. Gen. 1:351. 1891.

Collection number:814

Synonyms: Scorzonera resedifolia L., Sp. PI. 1198. 1753; Leontodon mucronatum Forsk., Fl. Egypt. 144. 1775; Launaeamucronata (Forsk.) Muschler, Man. Fl. Egypt 2:1057. 1912;L. resedifolia var. pulchella Pamp., Agriculture Col. 22:366 t. 1 verson f. 2. 1928; I.c. 480; Keith. I.c. 599.

Distribution: N. Africa, Spain and Iran. - In Tarhuna: Msabha, Terghlat and Wlaad Hammad. 
Fl.March-May.

Collectors: MSB

Collection date: 19.05.2016

5.32. Leontodon simplex (Viv.) Widderin Phyton 12:209. 1967.

Collection number: 911

Synonyms: Hieracium simplex Viv., Fl. Lib. Spec. 50 tab. 1824. Leontodon muelleri (Schultz) Fiori in Fiori \& Paol. , Fl. Anal. It. 3:396. 1904.

Distribution: N. Africa and Europe. - In Tarhuna: Terghlat.

FI.February-June.

Collectors: MSB

Collection date: 12.06 .2017

5.33. Nolletia chrysocomides (Desf.) Cass.,Dict. Sci. Nat. 37:479. 1825.

Collection number: 831

Synonyms: Conyza chrysocomides Desf., Fl. Atl. 2:269. T. 232. 1798.

Distribution: N. Africa; (Morocco to Libya); S. Spain. - In Tarhuna: Msabha.

FI. January- May.

Collectors: MSB

Collection date: 19.05 .2016

5.34. *Onopordeum arenarium (Desf.) Pomel, Nouv. Mat. FI. AtI. 20. 1874.

Collection number: 815

Synonyms: Carduus arenarius Desf., Fl. AtI. 2:247 t. 222. 1799; Onopordum sibthorpianum Boiss. \& Heldr. var. arenarium (Desf.) Durand \& Barratte. FI. Lib. Prodr. 141. 1910.

Distribution: N. Africa (Libya, Tunisia, Algeria). In Tarhuna: Msabha and Terghlat.

FI.May-June.

Collectors: MSB

Collection date: 19.05 .2016

5.35. *Phagnalon rupestre (L.) DC., Prodr. 5:396. 1836.

Collection number: 737

Synonyms: Conyza rupestrisL., Mantissa 113.1767.

Distribution: Mediterranean region\& S. Spain.

- In Tarhuna: Khsaya, Msaaed, Msabha, Sok ElAhedand Whedat.

FI. February-May.

Collectors: MSB

Collection date: 22.03 .2016

5.36. *Picris asplenoides L., Sp. PI. 793. 1753. Collection number: 805

Synonyms: Crepis radicata Forsk., Fl. Aegypt. 145. 1775; Picris Iyarata Delile, Desct. Egypt Hist.
Nat. 259. 1813; P. radicata (Forsk.) Less., Syn. Gen. Comp. 134. 1832.

Distribution: N. Africa. - In Tarhuna: Arabeen, Msaaed, and Wlaad Hammad.

FI. January-May.

Collectors: MSB

Collection date: 19.05 .2016

5.37. Reichardia tingitana (L.) Roth, Bot. Abh. 35.1787 .

Collection number: 785

Synonyms: Scorzonera tingitanaL., Sp. PI. 791. 1753; Picridium tingitanum (L.) Desf., Fl. Atl. 2: 220. 1799; Sonchus tingitanus (L.) Lam., Encycl. Meth. Bot. 3:397.1792.

Distribution: N. Africa, Canary Islands, Spain, Portugal, Greece, Arabia, N. Iraq, Iran, Afghanistan andIndia. - In Tarhuna: Dawoon and Zurgan.

FI. April-June.

Collectors: MSB Collection date: 8.04.2016

5.38. *Rhanterum suaveolens Desf., Fl. Atl. 2:291. 1799.

Collection number: 883

Distribution: N. Africa. - In Tarhuna: Whedat and Wlaad Hammads.

Fl:March-May.

Collectors: MSB

Collection date: 10.05.2017

5.39. Scorzonera undulata Vahl, Symb. Bot. 2:86. 1791.

Collection number: 695

Synonyms: Scorzonera serrulata Viv., Fl. Lib. Spec. 49. T. 17.1824; S. undulate var. alexandrina (Boiss.) Barratte in Bonnet \& Barratte, Cat. Rais. PI. Vas. Tunisie 262. 1896; S. undulate ssp. alexandrina (Boiss) Maire, in Jahandiez \& Maire, Cat. PI. Moroc. 3:839. 1934.

Distribution: Circum-Mediterranean region. - In Tarhuna: Dawoon, Mckalef, Meghawshaand Terghlat.

FI. March-May.

Collectors: MSB Collection date:19.03.2016

5.40. Senecio gallicus Chiax in Vill., PI. Dauph. 1:371. 1786

Collection number: 696

Synonyms:Senecio desfontaineiDruce, List Brit. PI. ed. 2, 61'. 1828; S. gallicus var. laxiflorus (Viv.) DC., Prodr. 6: 346. 1838.

Distribution: Mediterranean element. - In Tarhu- 
na: Dawoon, Meghawsha, Shersharah and Terghlat.

FI. January-April.

Collectors: MSB

Collection date: 19.03 .2016

5.41. * Sonchus asper (L.) Hill. Brit. 1:47 t 34. 1769. Collection number: 753

Synonyms: Sonchus oleraceus var. asper L.,Sp. PI. 794.1753.

Distribution: Europe, N. Africa, Sudan, Ethiopia and W. Asia. - In Tarhuna: Hejaz.

FI. November-January.

Collectors: MSB Collection date:22.03.2016

5.42. Sonchus oleraceus L., Sp. PI. 794. 1753.

Collection number: 723

Synonyms: Sonchus ciliatusLam.,Fl. Fr. 2:87. 1778; S. lacerus Willd., Sp. PI. ed.4.3:1513.1803;S. oleraceus var. lacerus (Willd.) Wallr.,Sched. Grit. 432. 1822; S.oleraceus ssp. lacerus (Willd.) Clos, in Bull. Soc. Bot. Fr. 48:59. 1901; S.oleraceus ssp. ciliatus (Lam.) Zenari, in Nuovo Giorn. Bot. Ital. 31: 5. 1924.

Distribution: Europe, N. Africa and N.W. Asia. - In Tarhuna: Mckhalef and Terghlat.

FI.Throughout the year.

Collectors: MSB Collection date: 19.03.2016

\section{Boraginaceae}

6.1.*Arnebia decumbens (Vent.) Coss. \& Kral. In Bull. Soc. Bot. Fr. 4:402. 1857.

\section{Collection number: 884}

Synonyms: Lithospermum decumbens Vent. Descr. PI. Jard. Cels. 37. 1801; Arnebia decumbens ssp. maccrocalyx (Coss. \& Kral.) Riedel in Rech. f. Fl. Iran. 48:160. 1967.

Distribution: N. Africa, S. Iran, and S. \& C. Asia. In Tarhuna: Arabeen and Wlaad Hammad.

FI. February-June.

Collectors: MSB Collection date: 10.05.2017

6.2. Echiochilon fruticosum Desf., Fl. Atl. 1:167. Tab. 47. 1798.

Collection number:786

Distribution: N. African element -Lebanon. - In

Tarhuna: Zurgan.

FI.February-June.

Collectors: MSB Collection date: 8.04.2016

6.3. Echium angustifolium Mill., Gard. Dict. ed. 8: 6. 1768.
Collection number: 754

Synonyms:Echium sericeum Vahl. Symb. 2:35. 1791; E. sericeum var. diffusum (Sibth. \& Sm.) Boiss., Fl. Or. 4:207.1879.

Distribution: Mediterranean region and Syria. - In Tarhuna: Dawoon, Hejaz, Khsaya, Msaaed, Msabha and Terghlat.

FI.January-March.

Collectors: MSB Collection date: 22.03.2016

6.4. Echium humile Desf., fl. Atl. 1:165. 1798.

Collection number: 787

Synonyms: Echium angustifolium var. humile de Coincy in Morot J. de Bot. 16:215. 1902; Echium pycnanthum ssp. humile (Desf.) Jan. \& Maire, Cat. PI. Mar. 605. 1934.

Distribution: N. Africa, Egypt and Southern Europe. - In Tarhna: Khsaya and Wlaade Hammad.

Fl.March-May.

Collectors: MSB Collection date: 8.04.2016

6.5. Echium sabulicola Pomel, Nouv. Mat. Fl. Atl. 90. 1874.

Collection number: 755

Synonyms: Echium confusum de Coincy ex Morot in J. de Bot. 14. 298.1900; Echium confusum var. decipiens (Pomel) de Coincy ex Morot in J. Bot. 16:230.1902.

Distribution: W. Mediterranean region and N. Africa. - In Tarhuna: Hejaz.

FI. March-April.

Collectors: MSB

Collection date:22.03.2016

6.6. Elizaldia calycina (Roem. \& Schultes) Maire, Bull. Soc. Hist. Nat. Afr. Nord 20: 192. 1929.

Collection number: 858

Synonyms: Lycopsis calycina Roem. \& Schultes Syst. 4:74.1819; Elizaldia violacea (Desf.) I.M. Johnst. ssp. calycina (Roem. \& Schultes) Maire, I.c. 1920.

Distribution.Mediterranean region. - In Tarhuna:Arebeen and Dawoon.

FI.January-June.

Collectors: MSB

Collection date: 27.04 .2017

6.7.*Heliotropium europaeum L. Sp.PI. 130. 1753. Collection number: 816

Synonyms: Heliotropium commutatum Roem. \& Schult. Syst. Veg. 4:219. 1819.

Distribution: Asia Minor, Central and South Europe, N. Africa, Iraq and Iran. - In Tarhuna: Msabha. 
FI.November-May.

Collectors: MSB

Collection date:19.05.2016

6.8* Lappula spinocarpos (Forsk.) Ascherson ex O. Kuntze in Acta Horti Petrop. 10: 215.1887. Collection number: 885

Synonyms: Anchusa spinocarpos Forsk. FI. Aegypt. -Arab. 41. 1775; Echinospermum spinocarpos (Forsk.) Boiss., Fl. Or. 4:249. 1879.

Distribution: N. Africa, Sinai, Palestine, Syria, Iraq, Iran, Afghanistan, Pakistan and Central Asia. - In Tarhuna: Wlaad Hmmad.

FI.January-May.

Collectors: MSB

Collection date: 10.05.2017

\section{Brassicaceae}

7.1. Brassica tournefortii Gouan, III. Olbs. Bot.44, t. 20A. 1773.

Collection number: 785

Synonyms: Brassica stocksii Hook. f. \& Thoms. In J. Linn. Soc. Bot. 5:171. 1861.

Distribution: N. Africa, S. Europe, estwards to Pakistan. - In Tarhuna: Arabeen, Msaaed, Sok-ElAhed and Zurgan.

FI.January - March

Collectors: MSB

Collection date: 8.03.2016

7.2. Didesmis bipinnatus (Desf.) DC.,Syst. 2: 659 . 1821.

Collection number: 756

Synonyms:Sinapis bipinnata Desf.; Fl. Atlant. 2:97. 1799; Rapistrum bipinnatum (Desf.) Coss. et Kral, in B. Soc. Bot. France 4:395. 1857.

Distribution: Egypt, Libya, Tunisia, Algeria. - In Tarhuna: Hejaz, Khsaya, Terghlat and Zurgan.

FI.February - April.

Collectors: MSB Collection date: 22.03 .2016

7.3. Diplotaxis muralis (L.) DC., Syst. Nat. 2: 634. 1821.

Collection number: 859

Synonyms: Sisymbrium muraleL., Sp. PI. 658. 1753.

Distribution: Europe and N. Africa. - In Tarhuna: Dawoon, Sok-El-Ahed and Terghlat.

FI.January - March.

Collectors: MSB Collection date: 27.04.2017

7.4. Enarthrocarpus clavatus Del. ex Godr., Fl. Juven. Adv. 4. 1853.

Collection number: 789

Synonyms: Enarthrocarpus trabalis Pomel,
Nouv, Mat. 358. 1875.

Distribution: N. Africa. - In Tarhuna: Msaaed and Zurgan.

Fl. February-April.

Collectors: MSB Collection date: 8.04.2016

7.5. Eruca sativa Mill., Gard. Dict. ed.8,1:1768.

Collection number: 697

Synonyms: Brassica eruca L, Sp. PI. 667. 1753; Eruca vesicaria (L.) Cav. ssp. sativa (Mill)Thell., adv. Montpell. 260. 1912, et var. sativa (Mill.) Thell. In Hegi, 111. Fl. Mittleur. 4 (1): 201. 1918.

Distribution: N. Africa. - In Tarhuna: Makhalefand Whedat.

FI.March- April.

Collectors: MSB Collection date: 19.03.2016

7.6. Hussonia pinnata (Viv.) Jafri comb. Nov. 1977.

Collection number: 860

Synonyms: Raphanus pinnatus Viv., Fl. Lib. Sp. 37. Tab. 16.1824; Hussonia uncata Boiss., Diagn. Ser. 1.8: 47. 1849.

Distribution: N. Africa, Sinai, Arabia Petraea. - In Tarhuna: Dawoon and Sok-El-Ahed.

Fl.March-May.

Collectors: MSB Collection date: 27.04.2017

7.7. Matthiola fruticulosa (L.) Maire in Jah. \& Maitre, Cat. PI. Maroc. 2: 311. 1932.

Collection number: 837

Synonyms:Cheiranthus fruticulosus L., Sp. PI. 662. 1753.

Distribution: Europe, N. Africa, Cyprus, Lebanon, Turkey. - InTarhuna: Terghlat and Zurgan.

FI.February. - April.

Collectors:MSB Collection date: 19.05.2016

7.8. Matthiola longipetala (Vent.) DC., Syst. 2:174.1821.

Collection number: 698

Synonyms: Cheiranthuslongipetalus Vent., Hort. Cals. 93. 1802; Matthiola oxyceras DC., I.c. 173.

Distribution: Irano-Turanian element: S. Europe, N.Africa, Arabia, W. Asia, to S. Russia \&Turkey. - In Tarhuna: Khsaya, Maghawsha, Sharshara, Sok-ElAhed and Zurgan.

FI.February-May.

Collectors: MSB

Collection date: 19.03 .2016

7.9. *Sinapis alba L., Sp. PI. 668. 1753.

Collection number: 872 
Synonyms: Brassica alba (L.) Rabenh., Fl. Lusatica, 1:154. 1893; S. alba ssp. mairei (Lindb.) Maire in M. C. 1365.1933.

Distribution: Europe, N. Africa, S.W. Asia. -In Tarhuna: Sok-El-Ahed.

FI.February-April.

Collectors: MSB

Collection date:3.05.2017

7.10. Sisymbrium irio L., Sp. PI. 659.1753.

Collection number: 790

Synonyms: Sisymbriumirioides Boiss. In Ann. Sc. Nat. Ser. 2. 17: 76. 1842.

Distribution: Europe, N.Africa, S.W. \& C. Asia. - In Tarhuna: Zurgan.

FI.February-May.

Collectors: MSB

Collection date:8.04.2016

8. Caesalpiniaceae

8.1. *Ceratonia siliqua L., Sp. PI. 1026. 1753. Collection number: 873

Distribution: S. Europe, Cyprus, N. Syria, N. Africa. - In Tarhuna:Sok-El-Ahed.

FI.January-November.

Collectors: MSB

Collection date: 3.05 .2017

\section{Caryophyllaceae}

9.1. Silene colorata Poiret, Voy. Barb. 2:163. 1789. Collection number: 757

Synonyms: Silene bipartite Desf., Fl. AtI. 1: 352. T. 100. 1798; S. ligulata Viv., FI. Lib. Spec. 24. 1. 12., f. 3. 1824.

Distribution: S. Europe, Turkey, Russia, N. Africa, Arabia and Pakistan. - In Tarhuna: Hejaz, Wlaad Hammad and Zurgan.

FI. January-June.

Collectors: MSB Collection date: 22.03.2016

9.2. *Silene gallica L., Sp. PI. 417. 1753.

Collection number: 886

Synonyms: Silene quinquevulneraL., Sp. PI. 416. 1753.

Distribution: N. Africa, Europe, Asia. - In Tarhuna: Wlaad Hammad.

FI.November-May.

Collectors: MSB

Collection date: 10.05 .2017

\section{Chenopodiaceae}

10.1. *Chenopodium album L., Sp. PI. 219. 1753.

Collection number: 806

Synonyms: None.

Distribution: Europe, Asia and N. Africa. - In Tarhuna: Msaaed.
FI.March-November.

Collectors: MSB Collection date: 19.05.2016

10.2. *Chenopodium murale L., Sp. PI. 219. 1753. Collection number: 791

Distribution: Cosmopolitan species. - InTarhuna: Msaaed, Terghlatand Zurgan.

FI.January -May.

Collectors: MSB Collection date: 8.04 .2016

10.3. *Hammada scoparia (Pomel) Iljin in J. Bot. US, 33: 583. 1943.

Collection number: 843

Synonyms:Haloxylon scoparium Pomel., Nouv. Mat. Fl. Atl. 335. 1875; Salsola articulate Cav. I.c. 3: 43.t. 284. 1794 Haloxylong articulatumssp. scoparium (Poml.) Batt. In B.\& T., Fl. Alg. 765. 1890; Arthrophytum scoparium. (Pomel.) lijin ex Jeh. \& Maire, Cat. Maroc. 975. 1914.

Distribution: S.E. Spain, N. Africa, Iran. - In Tarhuna: Khsaya, Sok-El-Ahed and Terghlat.

Fl.October-May.

Collectors: MSB Collection date:27.04.2017

\section{Cictaceae}

11.1. *Fumana arabica (L.) Spach in Ann. Sc. Nat. Ser. Il. 6: 359. 1836.

Collection number: 844

Synonyms: Cistusarabicus L., Sp. PI. ed. 2: 745. 1762; Helianthemum arabicum (L.) Pers., Syn. PI. 2: 80. 1807.

Distribution: N. Africa, Italy, Turkey, Syria and Iran. - In Tarhuna: Khsaya.

FI. March-April.

Collectors: MSB

Collection date: 27.04 .2017

11.2. *Fumana thymifolia (L.) Spach ex Webb, Iter, Hisp. 69. 1838.

Collection number: 817

Synonyms:Cistus thymifolius L., Sp. PI. 528. 1753;Fumana viscidaSpach,Hist. Veg. Phan. 6: 12. 1838F. viscid var. thymifolia (L.) Spach inSpach in Ann. Sc. Nat. Bot. Ser. 2. 6: 359.1836.

Distribution: S. Europe, N. Africa to W. Syria \& Turkey. - In Tarhuna: Msaaed and Terghlat.

FI.March - April.

Collectors: MSB Collection date: 19.05.2016

11.3. Helianthemum ciliatum (Desf.) Pers., Syn. PI. 2: 79. 1806.

Collection number: 807

Synonyms: Cistus ciliates Desf., Fl. Atlant. 1: 421. 
Tab. 109. 1798.

Distribution: Spain, N. Africa, Egypt, Palestine and Arabia Petrea. - In Tarhuna: Msaaed.

FI. March-April. Collectors: MSB

Collection date: 19.05 .2016

11.4. Helianthemum hirtum (L.) Mill., Gard. Dict. ed. 8, no. 14.1768 .

Collection number: 699

Synonyms: Cictus hirtusL., Sp. PI. 528. 1753; Helianthemum ruficosum (Viv.) Spreng., Spet. 2: 589. 1825; Helianthemumhirtum ssp.ruficosum (Viv.) Maire in Jahand. Maire, Cat. PI. Maroc. 900.1934. Distribution: S.W. Europe, Algeria, Mauritania and Liby. - In Tarhuna: Hejaz, Meghawsha and Terghlat.

FI. March-April.

Collectors: MSB

Collection date: 19.03 .2016

11.5. *Helianthemum kahiricum Delile, Fl. Egypt 237. Tab. 31. 1813.

Collection number: 818

Synonyms: Helianthemum libycum Pomel, Nouv. Mat. Fl. Atl. 352. 1875.

Distribution: N. Africa, Egypt, Palestine and Arabia Petrea. - In Tarhuna: Msaaed.

FI. March-April.

Collectors: MSB Collection date: 19.05.2016

11.6. *Helianthemum lavandulifolium Miller, Gard. Dict. ed. 8 no, 13.1768.

Collection number: 724

Synonyms: HelianthemumracemosumPers., Sy. 2: 79.1819.

Distribution: Europe and N. Africa. - In Tarhuna: Mckhalef.

FI. February-April.

Collectors: MSB

Collection date: 19.03 .2016

11.7. *Helianthemum lippii (L.) Dum. Cours.,Le Bot. Cult. 3: 130. 1802.

Collection number: 865

Synonyms: Cistus lippiiL., Mantissa 2: 245. 1767.

Distribution: N. Africa, Arabia, W. Asia- E. Pakistan. - In Tarhuna: Sharshara and Sok-El-Ahed.

FI. March-May.

Collectors: MSB Collection date:27.04.2017

11.8. *Helianthemum stipulatum (Forsk.) C. Chr. In Dansk. Bot. Ark. 4 (3): 20. 1922.
Collection number: 838

Synonyms: Cistus stipulates Forsk., FI. Aeg. Arab, 101. 1775; Cistus ellipticum Desf., Fl. Atl. 1: 418. T. 107. 1800; Helianthemum ellipticum (Desf.) Pers. Synops. 2: 78. 1807.

Distribution: N. Africa, Greece, Anatolia, Egypt, Arabia and Iraq. - In Tarhuna: Terghlat.

FI. March-April.

Collectors: MSB Collection date: 19.05.2016

\section{Convolvulaceae}

12.1. Convolvulus althaeoides L., Sp. PI. 1: 156. 1753.

Collection number: 700

Distribution: S. Europe, Canary Islands \& Mediterranean region. - In Tarhuna:Hejaz, Mckhalef, Meghawsha and Sok-El-Ahed.

FI.March-June.

Collectors: MSB Collection date: 19.03.2016

12.2. * Convolvulus arvensis L.,Sp. PI. 153. 1753. Collection number: 845

Distribution: Temperate regions throughout the world except Australia. - In Tarhuna: Khsaya, Shersharah, and Terghlat.

FI.March-August.

Collectors: MSB

Collection date: 27.04 .2017

12.3. *Convolvulus dorycnium L., Syst. Nat. ed. 10, 2:923. 1759.

Collection number: 832

Distribution: Greece, Crete, Tunisia, Libya and Egypt. - In Tarhuna: Msabha.

FI.????

Collectors: MSB Collection date:19.05.2016

12.4. Convolvulus supinus Coss. et Kral. In Bull. Soc. Bot. Fr. 4:400. 1857.

Collection number: 792

Distribution: N. Africa (Libya, Tunisia, Algeria, Morocco). - In Tarhuna: Terghlat.

Fl.April-June.

Collectors: MSB Collection date: 8.04 .2016

\section{Crasulaceae}

13.1. Sedum sediforme (Jacq.) Pau. In Act. Mem. Prim. Congr. Nat. Esp. Zaragoza 246. 1909.

Collection number: 725

Synonyms: Sempervivum sediforme Jacq., Hort., Vindob. 1:35. T. 81. 1770; Sedum altissimum Poiret 
inLamk. Encycl. 4, 634. 1796.

Distribution: S. Europe, Mediterranean region \& W. Asia. - In Tarhuna: Hejaz and Mckhalef.

FI. March-July.

Collectors: MSB Collection date: 19.03.2016

\section{Cucurbitaceae}

14.1. *Bryonia cretica L., Sp. PI. 1013. 1753.

Collection number: 846

Synonyms: Bryonia dioica Jacq., Fl. Austr. 2: 59. T. 199. 1774; B. cretica ssp. dioica (Jacq.) Tutin in Feddes Report. 79: 61. 1968.

Distribution: Europe, N. Africa and W. Asia. - In Tarhuna: Khsaya.

FI.February- April.

Collectors: MSB

Collection date: 27.04 .2017

\section{Cuscutaceae}

15.1. Cuscuta planiflora Ten., FI. Nap. 3: 250, tab. 220.1829.

Collection number: 758

Synonyms: Cuscuta sicula Ten. ex Englem. In Trans. Acad. St. Louis 1: 466. 1859.

Distribution: Mediterranean region, N. Africa, Mauretaniaand Iran. - In Tarhuna: Hejaz.

FI. March-May.

Collectors: MSB Collection date: 22.03.2016

\section{Dipsacaceae}

16. 1. Scabiosa arenaria Forskal,Fl. Aeg-Arab. LXI no. 89.1775.

Collection number: 701

Synonyms: Scabiosa rhizantha Viv., FI. Lib. Spec. 6. t. 3. 1824; Asterocephalus arenarius (Forsk.) Vis., Ic. PI. Quar. Aeg. -Nub. 6. t. 1.1.1836.

Distribution: N. Africa, Syria and Palestine. - In Tarhuna: Hejaz, Msaaed and Terghlat.

FI. March-May.

Collectors: MSB Collection date: 19.03.2016

\section{Ephedraceae}

17.1. Ephedra altissima Desf., Fl. Atl. 2:372, 1799;Pamp. Trip. 5.1914; Mair, Fl. Af. Nord. 1:145. 1952; Keith, Prelim. Check list Lib. 1.456. 1965.

Collection number: 901

Distribution: N. Africa. - In Tarhuna: Arbeen.

FI. March-May.

Collectors: MSB

Collection date: 10.05 .2017

18. Euphorbiaceae
18.1. *Euphorbia exigua L., Sp. PI. 456. 1753.

Collection number: 819

Synonyms: Euphorbia exigua var. retusa L., Sp. PI. 45. 1753.

Distribution: Europe, N. Africa, Canaries \& W. Asia. - In Tarhuna: Msabha.

FI. February-May.

Collectors: MSB Collection date: 19.05.2016

18.2. *Euphorbia falcata L., Sp. PI. 456. 1753.

Collection number: 793

Synonyms: Euphorbia acuminateLam., Encyc. 2: 426. 1788; E. falcate var. acuminate (Lam.) S. Amand, Fl. Agen. 189, 1821.

Distribution: N. Africa, Pakistan and India. - In Tarhuna: Zurgan.

FI. February-June.

Collectors: MSB Collection date: 8.04.2016

18.3. Euphorbia helioscopia L., Sp. PI. 459.1753. Collection number: 874

Synonyms: Euphorbia helioscopioides Loscos \& Pard., Inconf. Arag 93. 1863.

Distribution: Europe, N. Africa to Asia. - In Tarhuna: Sok-El-Ahed.

FI. January-May.

Collectors: MSB

Collection date: 3.05 .2017

18.4. Euphorbia terracina L., Sp. PI. ed. 2: 654. 1762.

Collection number: 808

Synonyms: Euphorbia trapezoidalis Viv., FI. Lib. Spec. 25, tab. 4. 1824.

Distribution: Europe, Mediterranean region, Canaries and Syria. - In Tarhuna:Msaaed.

FI. January-June.

Collectors: MSB Collection date: 19.05.2016

18.5. *Ricinus communs L., Sp. PI. 1430. 1753.

Collection number: 820

Distribution: MostTropical and subtropical countries. - In Tarhuna: Msabha and Terghlat.

FI.Throughout the year

Collectors: MSB Collection date: 19.05.2016

19. Fabaceae

19.1. *Anthyllis tetraphylla L., Sp.PI. 719. 1753.

Collection number: 759

Synonyms: Physanthyllis tetraaphylla (L.) Boiss., Voy. Bot. Espagne 2: 162. 1839 
Distribution: Mediterranean region. - In Tarhuna: Hejaz.

FI. February-April. Collectors: MSB

Collection date: 22.03 .2016

19.2. *Argyrolobium uniflorum (Decne.) Jaub. \& Spach, III. PI. Or. 1: 115. 1843.

Collection number: 760

Synonyms:Cystisus uniflorus Decne. In Ann. Sc. Nat. Bot. Ser. II. 3: 265. 1835.

Distribution: N. Africa, Sinai, Palestine, Syria, S. Anatolia. - In Tarhuna: Hejaz, Msabha, Terghlat and Zurgan.

FI.March-April.

Collectors: MSB

Collection date: 2 2.03.2016

19.3. *Astragalus asterias Stev. ex Ledeb., Fl. Ross. 1: 636. 1843.

Collection number: 809

Synonyms: Astragalus aristidis Coss. ex Batt. Et. Trab., Alg. 256. 1889.

Distribution: C. \& W. Asia, Arabia and N. Africa. In Tarhuna: Msaaed.

Fl.January-March.

Collectors: MSB Collection date: 19.05.2016

19.4. *Astragalus caprinus L., Sp. Pl. ed. 2: 1071. 1763.

Collection number: 847

Synonyms: Astragalus lanigerus Desf., Fl. Atl. 2: 181. t. 202. 1799. A. caprinus ssp. lanigerus (Desf.) Maire in Jehand. \& Maire, Cat. PI. Maroc. 2:413. 1932.

Distribution: N. Africa, Palestine, Turkey, Cyprus, Syria, Iraq andlran. - In Tarhuna: Khsaya.

Fl. Almost throughout the year.

Collectors: MSB Collection date: 27.04.2017

19.5. *Astragalus sinaicus Boiss., Diagn. Ser. 1.9. 57. 1849.

Collection number: 761

Distribution: Greece, Aegean, Crimea, Turkey, N. Africa, Sinai and Arabia. - In Tarhuna: Hejaz and Wlaad Hammad

FI. March-May.

Collectors: MSB Collection date:22.03.2016

19.6. *Astragalus stella Gouan. I11. Obs. Bot. 1773. Collection number: 738

Synonyms: Astragalus acutifolius DC. ex Steud.
Nom. 88. 1821; Stella radiate Fourr. In Ann. Soc. Linn. Lyon 16: 364. 1863.

Distribution: Mediterranean region \&Arabia. - In Tarhuna: Khsaya and Whedat.

FI. February-April.

Collectors: MSB Collection date:22.03.2016

19.7. Astragalus tribuloides Del., Fl. Aeg. I11. 70. 1813.

Collection number: 726

Synonyms: Oxyglottis tribuloides (Delile) Nevski in Acta Inst. Bot. Acad. Sc. URSS ser1 (4): 259. 1937.

Distribution: N. Africa, Palestine, Turkey, W. Asia, Arabia. - In Tarhuna: Mckhalef.

FI. March-April.

Collectors: MSB

Collection date: 19.03 .2016

19.8. *Astragalus trigonus DC., Astragal. 81. 1802.

Collection number: 762

Synonyms: Astragalus leucacanthus Boiss., Diagn. Ser. 1.9: 93.1849; A. trigonus var. leucacanthus (Boiss.) Tackh. et Boulos in Pub. Cairo Univ. Herb. 5:69. 1972.

Distribution: Egypt and Libya. - In Tarhuna: Hejaz. FI. March-April.

Collectors: MSB Collection date: 22.03.2016

19.9. * Calicotome villosa (Poir.) link in Schrader, Nau. J. Bot. 2(2): 51. 1806.

Collection number: 763

Synonyms:Spartium villosum Poir., Voy. Barb. 2: 207. 1789; Calicotomerigida (Viv.) Maire \& Weil., in Bull. Soc. Hist. Nat. Afr. Nord 30: 271. 1939.C. villosa var. rigida (Viv.) Beg. et Vacc., Cont. Fl. Lib. 47. 1912.

Distribution: S. Europe, N. Africa, Palestine. - In Tarhuna: Hejaz, Khsaya and Terghlat.

Fl.January-April.

Collectors: MSB Collection date:22.03.2016

19.10. *Coronilla scorpioides (L.) Koch, Syn. Fl. Germ. Helv. 188. 1853.

Collection number: 764

Synonyms: Ornithopus scorpioidesL., Sp. PI. 744. 1753

Distribution: Europe, N. Africa, Palestine, Turkey, Syria and S. Iran. - In Tarhuna: Hejaz, Khsaya and Shersharah. 
FI. February- April.

Collectors: MSB

Collection date: 22.03 .2016

19.11. Hippocrepis bicontorta Loisel, Nouv. Not. 32. 1827.

Collection number: 765

Synonyms: Hippocrepis conigeraBoiss, Diagn. Ser. 1.2: 102. 1843..

Distribution: N. Africa, Sinai, Palestine, Arabia, Kuwait, Bahrain and S. Iran. - In Tarhuna: Arabeen, Hejaz, and Terghlat.

FI. March-May.

Collectors: MSB Collection date: 22.03.2016

19.12. *Hippocrepis ciliata Willd. In Ges. Naturf. Fr. Berlin Mag. 2: 137. 1808.

Collection number: 766

Distribution: Mediterranean region. - In Tarhuna: Hejaz.

FI. February-April.

Collectors: MSB Collection date:22.03.2016

19.13. *Hippocrepis cyclocarpe Murb. In Act. Univ. Lund 33(12):80.1897.

\section{Collection number: 767}

Synonyms: Hippocrepis cyclocarpe var. pubescens Pamp. in Bull. Socl. Bot. Ital. 14.1914.

Distribution: Egypt, Libya and Tunisia. - In Tarhuna: Hejaz.

FI. March-May.

Collectors: MSB Collection date:22.03.2016

19.14. *Hymenocarpos circinatus (L.) Savi, Fl. Pis. 2:205. 1798.

Collection number: 768

Synonyms: Mddicago circinataL., Sp. PI. 778. 1753; Corniciana circinata (L.) Boiss., Voy. Bot. Esp. 2: 163. 1839.

Distribution: S. Europe, N. Africa and Asia. - In Tarhuna: Hejaz.

FI. Marc-April.

Collectors: MSB Collection date:22.03.2016

19.15. Lotus cytisoides L., Sp.PI. 776. 1753.

Collection number: 769

Synonyms: Lotus cretius var. cytisoides (L.) Boiss., Fl. Or. 2:165. 1872; L. cretius ssp. Cytisoides (L.) Aschers.

Distribution: Mediterranean region. - In Tarhuna: Terghlat.
FI. March-June.

Collectors: MSB Collection date:22.03.2016

19.16. Lotus edulis L., Sp. PI. 774. 175.

Collection number: 770

Distribution: Mediterranean region. - In Tarhuna: Hejaz.

FI. March-May.

Collectors: MSB Collection date: 22.03.2016

19.17. *Lotus halophilus Boiss. et Spruner in Boiss., Diagn. Ser. 1.2.1843.

Collection number: 771

Synonyms: Lotus pusillus Viv., Fl. Lib. Spec. 47, 1824; L. halophilus var. macranthus Boiss., Fl. Or. 2:17. 1872.

Distribution:E. Mediterranean, Greece, Italy, Crete, Sicily, Cyprus, N. Africa, Palestine, Turkey and W. Syria. - In Tarhuna: Targhlat.

FI.January-April.

Collectors: MSB Collection date:22.03.2016

19.18. *Medicago littoralis Rohde ex Lois., Not. Fl. France. 118. 1810.

Collection number: 772

Distribution: Mediterranean region. - In Tarhuna: Hejaz and zurgan.

FI. February-June.

Collectors: MSB Collection date:22.03.2016

19.19. *Medicago minima (L.) Bart., Cat. Piant. Siena 61.1776.

Collection number: 866

Synonyms: Medicago polymorpha var. minimaL., Sp. Pl. 780. 1753.

Distribution: Eurasia. Mediterranean region and S. Africa. - In Tarhuna: Shersharah.

Fl. February-April.

Collectors: MSB Collection date: 27.04.2017

19.20. *Medicago polymorpha L., Sp. PI. 779. 1753.

Collection number: 739

Synonyms:Medicago nigra Krock., Fl. Siles. 2 (2): 244. 1826; M. hispida Gaertn., Fruct. et Sem. 2:349. 1791.

Distribution: Mediterranean region, W, \& C. Asia. - In Tarhuna: Dawoon, Shersharah, Whedat and Wlaad Hammad.

FI. February-May. 
Collectors: MSB

Collection date: 2 2.03.2016

19.21. *Medicago tornata (L.) Mill., Gard. Dict. ed. 8. No. 3. 1768 .

Collection number: 702

Synonyms: Medicago polymorpha var. tornataL., Sp. PI. 780. 1753.

Distribution: Mediterranean region. - In Tarhuna: Mackhalef and Zurgan.

FI. February-May. Collectors: MSB

Collection date: 19.03 .2016

19.22. *Medicago truncatula Gaertn., Fruct. et Sem. 2:350. 1791.

Collection number: 773

Synonyms: Medicago tribuloides Desr. In Lam. met. 3: 635. 1792; Medicago tribuloides var. truncatula(Gaertn.) Koch, Syn. FI. Germ. Helv. !62. 1835.

Distribution: Mediterranean and Saharo-Arabian region to Syria. - In Tarhuna: Hejaz.

FI. February-May.

Collectors: MSB

Collection date: 22.03 .2016

19.23. * Ononis reclinata L., Sp. PI. ed. 2:1011.1753. Collection number: 703

Synonyms: Ononis mollis Savi, Mem, Mod. 9:351. t. 8. 1801; O. reclinata var. minor Moris, FI. Sard. 1:422. 1837; O. reclinata.var. mollis (Savi) Heldr. in Bull. Herb. Boiss. 6:292. 1808.

Distribution: Mediterranean region \& S.W. Asia. In Tarhuna: Meghawsha.

FI. March-June.

Collectors: MSB Collection date: 19.03.2016

19.24. Ononis sicula Guss., Cat. PI. Boccad. 78. 1821.

Collection number: 704

Synonyms: Ononis viscose L. var. sicula (Guss.) Fiori, Fl. Anal. Ital. 1:826. 1925.

Distribution: Mediterranean region (S. Europe, N. Africa) Arabia, Iraq and Iran. - In Tarhuna: Meghawsha.

FI. March-May.

Collectors: MSB

Collection date: 19.03 .2016

19.25. *Psoralea bituminosa L., Sp. PI. 763. 1753. Collection number: 705

Synonyms: Psoralea palestina Gouan, I11. Obs. Bot. 5. 1773; Aspalthium bituminosum (L.) Medik., Phil. Bot. 1: 211. 1789.

Distribution: S. Europe, N. Africa, Palestine, W.
Syria and Turkey. - In Tarhuna: Meghawsha.

FI. March-June.

Collectors: MSB Collection date: 19.03.2016

19.26. *Retama raetam (Forsk.) Webb in Webb \&berth., Phyt. Canar. 2:56.1842.

Collection number: 706

Synonyms:Genista raetam Forsk., Fl. Aeg. -Arab. 214. 1775; Spartium raetam (Forsk.) Spach in Ann. Sc. Nat. Ser. 2:19. 288.t. 16. 1843; Retama duriaei (Spach) Webb Spach in Ann. Sc. Nat. Ser. 2.20: 279.1843.

Distribution: N. Africa, Palestine and Syria. - In Tarhna: Khsaya, Meghawsha, Msabha, Sok-ElAhed, Terghlat, Whedat and Zurgan.

FI. February-June.

Collectors: MSB Collection date: 19.03 .2016

19.27. *Scorpiurus muricatus L., Sp. PI. 745. 1753.

Collection number: 707

Synonyms: Scorpiurus subvillosus L., Sp. PI. 745. 1753.

Distribution: Mediterranean region, Canaries, S. Iran and E. Afric a.- In Tarhuna: Mckhalef, Meghawsha, Sok-El-Ahed and Whedat.

FI. March-April.

Collectors: MSB Collection date: 19.03.2016

19.28. *Vicia ervilia (L.) Willd., Sp. PI. 3:1103. 1802. Collection number: 861

Synonyms: Ervum ervilia L., Sp. PI. 738. 1753.

Distribution: Mediterranean area, S.W. Asia. - In Tarhuna: Terghlat.

FI.February-June.

Collectors: MSB Collection date: 27.04.2017 19.29. *Vicia monantha Retz., Obs. Bot. 3:39. 1783.

Collection number: 912

Synonyms: Vicia calcarata Desf., Fl. Atlant. 2:166. 1798.

Distribution: Mediterranean area, S.W. Asia. - In Tarhuna: Terghlat.

FI.March-May.

Collectors: MSB Collection date: 12.06 .2017

19.30. *Vicia villosa Roth, Tent. Fl. Germ. 2:182. 1789.

Collection number: 867

Synonyms: Vicia reuteriana Boiss. \& Buhse, Aufz. 73. 1860; V. ambigua Guss., Prodr. 2:435. 1828; 
V. pseudo-caracca var. ambigua (Guss.) Durand \& Barratte, I.c. 876.

Distribution: S. \& C. Europe, N. Africa, Palestine, Turkey, S. \& W. Asia. - In Tarhuna: Shersharah.

FI. January-April.

Collectors: MSB Collection date:27.04.2017

20. Fumaraceae

20.1. Fumaria densiflora DC., Cat. Hort. Monspel. 113.1813.

Collection number: 794

Synonyms: Fumaria micrantha Lag., Elench. Hort. Matrit. 21. 1816; F. bracteosa Pomel, Nouv. Mat. 239. 1874.

Distribution: W. \& S. Europe, N. \&S. Africa, Turkestan and New Zealanda. - In Tarhuna: Msaaed and Zurgan.

FI. February-April.

Collectors: MSB Collection date: 8.04.2016

20.2. *Fumaria parviflora Lam., Encycl. 2:567. 1788.

Collection number: 875

Synonyms: Fumaria caspitosa Loscos ex Willk. \& Lange, Prod. Fl. Hisp. 3: 884. 1880; F. glauca Jord., Pugill. 8. 1852.

Distribution: Europe, S. Russia, N. Africa and S.W. Asia. - In Tarhuna: Sok-El-Ahed.

FI. February-April.

Collectors: MSB

Collection date:3.05.2017

\section{Geraniaceae}

21.1. *Erodium glaucophyllum (L.) L'Herit in Ait. Hort. Kew 2:416. 1789.

Collection number: 862

Synonyms: Geranium glaucophyllumL., Sp.PI. 679. 1753; E. tordilioides Viv., Fl. Lib. Spec. 39. 1824.

Distribution: N. Africa, Jordan, Sinai, Syria, Iraq, Kuwiat, Arabia and Iran. - In Tarhuna: Dawoon and Sok-El-Ahed.

FI. November-May.

Collectors: MSB

Collection date: 27.04 .2017

21.2. *Erodium hirtum (Forsk.) Willd., Sp. PI. 3:632. 1880.

Collection number: 902

Synonyms: Geranium hirtum Forsk., Fl. Aeg-Arab. 123. 1775.

Distribution: N. Africa, Jordan, Sinai, Palestine, Lebanon, Syria and Iraq. - In Tarhuna: Arabeen.
Fl. January-May.

Collectors: MSB

Collection date: 10.05 .2017

21.3. Erodium laciniatum (Cav.) Willd., Sp. PI. 3: 633. 1800.

\section{Collection number: 708}

Synonyms:Geranium laciniatumCav., Monad. Class. Diss. Dec. 4:228. t. 113. 1787; E. laciniatumssp. pulverulentum (Cav.) Burtt \& Lewis in Kew Bull. 1954. 405. 1954.

Distribution: N. Africa, S. Europe, Eastwards to Pakistan. - In Tarhuna: Arabeen, Meghawsha and Terghlat.

FI. January-May.

Collectors: MSB

Collection date:19.03.2016

21.4. *Erodium neuradifolium Delile in Godron, Fl. Juv. 17. 1853.

Collection number: 795

Synonyms: Erodium aegyptiacum Boiss., Diagn. PI. Or. Nov. 2 (3): 111. 1854; E. angulatum pommel Nouv. Mat. FI. Atl. 341. 1874.

Distribution: Canaries, S. W. Europe, N. Africa, Iran and Afghanistan. - In Tarhuna: Zurgan.

FI. December-June.

Collectors: MSB Collection date: 8.04.2016

\section{Hypecoaceae}

22.1. *Hypecoum geslini Coss. et Kral. In Bull. Soc. Bot. France 4: 522. 1857.

Collection number: 913

Synonyms: None.

Distribution: N. Africa (Egypt to Morocco). - In Tarhuna: Terghlat.

FI.May - June.

Collectors: MSB

Collection date:12.06.2017

23. Illceberaceae

23.1.*Gymnocarpos decander Forsk., Fl. Aegypt.Arab. 65. 1775.

Collection number: 709

Synonyms:Trianthema fruticosa Vahl, Symb. 1:32. 1790; Gymnocarpos fruticosum (Vahl) Pers., Syn. 1:262. 1805.

Distribution: Canary Islands estwards to Pakistan and Afghanistan through N. Africa and Arabia. - In Tarhuna: Meghawsha, Msabha, Terghlat and Whedat.

FI.February-May.

Collectors: MSB Collection date:19.03.2016

23.2. *Herniaria hemistemon J. Gay in Duch., 
Bot. 2:371. 1847.

Collection number: 710

Synonyms: Herniaria fruticosa Linn. Var. hemistemon (J. Gay) Barratte in B. \&B., Cat. Tun 65. 1896.

Distribution: N. Africa, Jordan, Iraq and Iran. - In Tarhuna: Meghawsha, Wlaad Hammad and Zurgan. FI. March-June.

Collectors: MSB Collection date:19.03.2016

23.3.*Paronychia arabica (Linn.) DC. In Lamk., Encycl. Method. Bot. 5:24.1804.

Collection number: 711

Synonyms: Illecebrum arabicumL., Mant. 1:51. 1767.

Distribution: N. Africa, Turkey, Palestine, Jordan, Syria, Lebanon, Iraq, Kuweit, Saudi Arabia and Iran. - In Tarhuna: Dawoon, Msabha, Shersharah, Sok-El-Ahed, Terghlat and Wlaad hammad.

FI.February -May.

Collectors: MSB Collection date:19.03.2016

23.4. *Paronychia chlorothyrsa Murb. In Acta Univ. Lund. 33:48. tab. 2. 1897.

\section{Collection number: 727}

Synonyms: Paronychiamacrosepala Sensu Ball. Journ. Bot. 204. 1875; Paronychia capitata (Linn.) Lamk. ssp. chlorothyrsa (Murb.) Maire et Weiller in Maire, Fl. Afr. Nord. 9:28. 1963.

Distribution: Widespread in Sahara from S. Libya to Morocco and Eritrea. - In Tarhuna: Hejaz, and Mckhalef.

FI. February- June.

Collectors: MSB

Collection date:19.03.2016

\section{Iridaceae}

24.1. Iris sisyrinchium L., Sp. PI. 40.1753; Viv., FI. Lib. Spec. 3.1824.

Collection number: 914

Synonyms:Moraea sisyrinchinum (L.) Ker-Gwal. In Kon. et Sims., Ann. 1:241. 1805; Iris syrtica Viv., FI. Lib. Spec. 3. 1824.

Distribution: S. Europe, N. Africa, S.W. Asia and Iran. - In Tarhuna: Terghlat.

FI.February-April.

Collectors: MSB Collection date:12.06.2017

\section{Lamiaceae}

25.1.*Ajuga iva (L.) Schreder, PI. Vert. Unilab, 25. 1773.
Collection number: 849

Synonyms: Teucrium iva L. Sp. PI. 563.1753.

Distribution: S. \& C. Europe, N. Africa, Syria, Palestine, andPakistan. - In Tarhuna: Khsaya and Sharsharah.

FI. March-April.

Collectors: MSB

Collection date:27.04.2017

25.2. Lavandula multifida L., Sp. PI. 572. 1753.

Collection number: 863

Distribution: N. Africa, Spain, Arabia, Syria, Plestine to Iran. - In Tarhuna: Dawoon, Sharsharahand Wlaad Hammad.

FI. March-May.

Collectors: MSB

Collection date:27.04.2017

25.3. *Marrubium alysson L., Sp. PI. 582. 1753.

Collection number: 876

Distribution: S. Europe, N. Africa (Mediterranean region), Syria, and Palestine. - In Tarhuna: Sok-ElAhed.

FI. January-May.

Collectors: MSB

Collection date:3.05.2017

25.4. * Marrubium vulgare L., Sp. PI.583. 1753.

Collection number: 848

Distribution: N. Africa, Europe, Caucasia, Turkey, Syria, Palestine. Iran \& Pakistan. - In Tarhuna: Khasya and Terghlat.

FI.April - July.

Collectors: MSB Collection date:27.04.2017

25.5. *Prasium majus L., Sp. PI. 601. 1753.

Collection number: 773

Distribution: Mediterranean and S. Aegean region. - In Tarhuna: Hejaz.

Fl. March-June.

Collectors: MSB Collection date:22.03.2016

25. 6. Rosmarinus officinalis L., Sp. PI. 23. 1753. Collection number: 833

Synonyms: Rosmarinus angustifolius Mill., Gard. Dict. ed. 8:1. 1758; Salvia Rosmarinus Schleid., Handb. Med.-Pharm. Bot. 1. 265. 1852.

Distribution: Portugal, N. W. Spain, Mediterranean region. - In Tarhuna: Msabha.

FI. Almost throughout the year.

Collectors: MSB Collection date:19.05.2016

25.7.Salvia lanigera Poir. In Lamarck, Encyclop. 
Method. Suppl. 5.149. 1817.

Collection number: 728

Synonyms: Salvia lanigera Desf., Tasb. L'ecol Mus. Hist. Nat. ed. 3:95. 1829.

Distribution: N. Africa, Siani, Cyprus, Palestine, Jordon, Syria, Lebanon, Arabia, Iraq and Iran. - InTarhuna: Terghlat.

FI. January- April.

Collectors: MSB

Collection date:19.03.2016

25.8. Salvia verbenacaL., Sp. PI. 25. 1753.

Collection number: 915

Synonyms:Salvia clandestineL., Sp. PI. ed. 2:36. 1762; Horminum verbenacea Mill., Gard. Dict. ed. 8, Horminum no. 1. 1768.

Distribution: Canary Islands, Maderia, N. Africa, Europe, Turkey and Iran. - In Tarhuna: Terghlat.

FI.December- April.

Collectors: MSB Collection date:12.06.2017

25. 9.*Scutellaria rubicunda Hornem., Hort. 2:968. 1815.

Collection number: 729

Synonyms: Scutellaria peregrine L. Sp. PI. 599. 1753.

Distribution: N. Africa, S. Balkan peninsula, C. \&

S. Italy and Sicily. - In Tarhuna: Mckhalef.

FI. March-May.

Collectors: MSB

Collection date:19.03.2016

25.10. *Teucrium polium L.,Sp. PI. 566. 1753.

Collection number: 868

Synonyms:Teucrium polium L. ssp. Cyrenaicum Maire et Weiller, Bull. Soc. Hist. Nat. Afr. Nord. 30:297. 1939.

Distribution: S. Europe, S. C. Russia, Turkey, Syria, Palestine, Saudi Arabia, Iraq, Iran, N. Africa,

E. Africa and Somalia. - In Tarhuna: Sharsharah and Terghlat.

Fl.March-July.

Collectors: MSB

Collection date:27.04.2017

25.11.*Thymus capitatus (L.) Hoffm. \& Link, Fl. Port. 1:123: 1809.

Collection number: 774

Synonyms:Satureja capitataL., Sp. PI. 568. 17753; Cordiothymus capitatus (L.) Rejchenb, f. in Oestr. Bot. WocherbI. XVIII, tab. 70.1857.

Distribution: Mediterranean region of Europe \& Africa. - In Tarhuna: Hejaz, Khsayaand Msabha.
FI. March-August.

Collectors: MSB

Collection date:22.03.2016

\section{Liliaceae}

26.1. *Asparagus stipularis Forsk., Fl. Aeg. -Arab. 72. 1775.

Collection number: 775

Synonyms: AsparagushorridusL.,f., Suppl. 203. 1781; A. stipularis var. brachyclados Boiss. Fl. Or. 5: 338.1884.

Distribution: N. Africa, Sicily, Cyprus, Palestine and Syria. - In Tarhuna: HejazandKhsaya.

FI.March-April.

Collectors: MSB Collection date:22.03.2016

26.2.*Asphodellus fistulosus L., Sp. PI. 309.1753 . Collection number: 712

Synonyms: Asphodellus fistulosus var. atlanticus Jah., Maire \& Weller in Maire, Fl. Afr. Nord., 5: 38. 1958.

Distribution: Mediterranean region. - In Tarhuna: Dawoon and Meghawsha.

FI. March-May.

Collectors: MSB Collection date:19.03.2016

26.3. Asphodellus microcarpus Salzm. \& Viv. In Viv., Fl. Cors. Diag. 5. 1824.

Collection number: 796

Synonyms: Asphodellus africanus Jord. In Bull. Soc. Bot. 7:730. 1860.

Distribution: Mediterranean region and Canaries. - In Tarhuna: Mdsabha and Zurgan.

FI. January-May.

Collectors: MSB Collection date: 8.04.2016

6.4. Dipcadi serotinum (L.) Medic. In Act. Acad. Theod. Palat. 6:431. 1790.

Collection number: 730

Synonyms: Hyacinthus serotinumL., Sp. PI. 317. 1753.

Distribution: S. Europe, Africa and Asia. - In Tarhuna: Mckhalef.

FI. F3ebruary-April.

Collectors: MSB

Collection date:19.03.2016

26.5. Muscari comosum (L.) Mill., Gard. Dict. ed. 8, no. 2. 1768.

Collection number: 713

Synonyms: Hyacinthus comosus L., Sp. PI. 318. 1753; Leopoldia comosa (L.) Parl., Fl. Palerm. 
1.438. 1845.

Distribution: S. Europe, N. Africa and W. Asia. - In

Tarhuna: Mckhalef, Sok-El-Ahed and Zurgan.

FI. March-June.

Collectors: MSB Collection date:19.03.2016

26.6. Scilla peruviana L., Sp. PI. 309. 1753.

Collection number: 740

Synonyms:Scilla

hemisphaerica

Boiss.,VoyageEspagne, 63, 1845; S. peruviana var. hipponensis (Jord. \& Forr.) Maire \& Weill. In M. C. 2842, 1939.

Distribution: N. Africa, Spain, Portugal and Italy. In Tarhuna: Hejaz, Terghlat and Zurgan.

FI. January- April.

Collectors: MSB Collection date:22.03.2016

26.7. Urginea maritima (L.) Baker in J. Linn. Soc. 13: 221. 1873.

Collection number: 877

Synonyms: Scilla maritimeL., Sp. L. 308. 1753; Urginea scillaSteinh. In Ann. Sc. Nat. Ser. 2.1:321. 1834.

Distribution: Mediterranean region and Canaries.

- In Tarhuna: Sok-El-Ahed.

FI. May- October.

Collectors: MSB

Collection date:3.05.2017

\section{Malvaceae}

27.1. *Lavatera bryoniifolia Miller, Gard. Dict. ed. 8. No.11. 1768.

Collection number: 887

Synonyms: Lavatera unguiculata Desf., Tabl. 145. 1804.

Distribution: Libya, Sicily, Greece, Aegean region, Turkey, Palestine. - In Tarhuna: Wlaad Hammad.

FI. May-June.

Collectors: MSB Collection date:10.05.2017

27.2. Malva parviflora L., Demonstr. PI. 18. 1753. Collection number: 797

Distribution: Libya, Mediterranean area, Syria, Arabia, Iran, Turkestan, Afghanistan and Pakistan.

- In Tarhuna: Terghlat and Zurgan.

FI. March-May.

Collectors: MSB Collection date: 8.04.2016

27.3. Malva sylvestris L., Sp. PI. 689. 1753.

Collection number: 714

Distribution: N. Africa, S. W. Asia, W. Europe. - In Tarhuna: Msaaed and Terghlat.
Fl.March-May.

Collectors: MSB

Collection date: 19.03 .2016

28. Papaveraceae

28.1. *Papaver hybridum L., Sp. PI. 506. 1753.

Collection number: 798

Distribution: Europe, N. Africa, S.W. \& C. Asia. - In Tarhuna: Khsaya.

FI. February-April.

Collectors: MSB Collection date: 8.04.2016

28.2. Papaver rhoea L., Sp. PI. 507. 1753.

Collection number: 850

Synonyms: Papaver chanceliae Maire, Annot. Fl. Alg. in B. S. H. N. Afr. Nord. 6:277. 1914. P. agrivagum Jord.,Diagn. 96. 1864.

Distribution: Europe, Asia and N. Africa. - In Tarhuna: Khsaya and Terghlat.

FI.January- June.

Collectors: MSB Collection date:27.04.2017

\section{Plantaginaceae}

29.1. Plantago albicans L., Sp. PI. 114. 1753.

Collection number: 799

Distribution: Spain, Portugal and Mediterranean region. - In Tarhuna: Dawoon, Msaaed, Msabha and Zurgan.

FI. February-June.

Collectors: MSB Collection date: 8.04 .2016

29.2. *Plantago amplexicaulis Cav., Icon. Descr. 2:22, Tab. 125. 1793.

Collection number: 731

Distribution: Spain, Algeria, Tunisia, Libya, Egypt, Arabia, Iran, Pakistan and W. India. - In Tarhuna: Hejaz, and Mckhalef.

FI. March- May.

Collectors: MSB Collection date:19.03.2016

29.3. Plantago coronopus L., Sp. PI. 115. 1753. Collection number: 741

Distribution: Europe, Mediterranean region, W. Asia. - In Tarhuna: Arabeen, Sok-El-Ahed, Whedat and Zurgan.

Fl. March-July.

Collectors: MSB

Collection date:22.03.2016

29.4. *Plantago lanceolata L., Sp. PI. 1753.

Collection number: 916

Synonyms: Plantago orientalis Stapf. In Denkschr. Akad. Wiss. Math. Nat. KI. 50:33. 1885. 
Distribution: N. Africa, Europe, S. Asia - the mountains of Tien Shan, Pamir Alaj and Australia. InTarhuna: Terghlat.

FI.March-July. Collectors: MSB

Collection date:12.06.2017

29.5. Plantago lagopus L., Sp. PI. 114. 1753.

Collection number: 742

Synonyms: Plantago lusitanica L., Sp. PI. ed. 2.1667.

Distribution: Mediterranean region to Afghanistan \&Pakistan. - In Tarhuna: Dawoon, Sok-El-Ahed and Whedat.

FI. March-May.

Collectors: MSB

Collection date: 22.03 .2016

29.6.*Plantago notata Lag. Gen. Sp. Nov. 7. 1816. Collection number: 821

Synonyms: Plantago syrtica Viv., Fl. Lib. Spec. 7.t. 3. 1824.

Distribution: S. Spain, N. Africa, Iraq, S. Iran and Transcaucasia. - In Tarhuna: Msabha.

FI. March-June.

Collectors: MSB Collection date:19.05.2016

30. Plumbaginaceae

30.1. *Limonium pruinosum (L.) O. Ktze, Rev. Gen. PI. 2: 396. 1891.

Collection number: 888

Synonyms: Statica pruinosa L. Mant. 59. 1767.

Distribution: N. Africa to Palestine and Syria. - In

Tarhuna: Wlaad Hammad.

Fl. October-May.

Collectors: MSB Collection date:10.05.2017

\section{Poaceae}

31.1. *Aegilops kotschyi Boiss., Diagn. PI. Or. Nov. Ser. 1, 7: 129. 1846.

Collection number: 743

Synonyms: Aegilops ovate var. triaristata acut. Non Willd. 1805.

Distribution: Egypt, Syria, Palistine, Jordan, Kuwait, Turkey, Cyprus and Iran. - In Tarhuna: Arabeen, Hejaz, Khsaya, Wlaad Hammad and Zurgan. FI. March-May.

Collectors: MSB

Collection date:22.03.2016

31.2. *Avena barbata Pott ex Link in Schrad., Journ. Fur Botanik, 2: 314. 1799.

Collection number: 715
Distribution: Mediterranean region, Anatolia, Iraq, Iran and Afghanistan. -In Tarhuna: Hejaz, Meghawsha and Msabha.

FI. January-May.

Collectors: MSB Collection date:19.03.2016

31.3. Bromus rigidus Roth in Bot. Mag. (Roem. \& Usteri) 4, 10: 21. 1790.

Collection number: 822

Synonyms: Bromus villosus Forsk. , Fl. Aeg. Arab. 23. 1775; B. villosus var. rigidus (Roth) Aschers. Et Graebn. In Syn. Mitt. Fl. 11: 596. 1901.

Distribution: Mediterranean region, Lebanon, Syria, C. \& S. Europe and S.W. Asia. - In Tarhuna: Khsaya.

FI. March-June.

Collectors: MSB Collection date:19.05.2016

31.4. *Bromus rubens L., Cent. PI. 1:5. 1755.

Collection number: 744

Synonyms: Anisantha rubens (L.) Nevski, in Acta Univ. As. Med. Ser. VIII b, Bot. Fsc. 17: 19. 1934.

Distribution: Europe, Mediterranean region, Anatolia, Iraq, Iran, Afghanistan and Turcomania. - In Tarhuna: Hejaz, Sok-El-Ahed, Whedat and Wlaad Hammad.

FI. March-May.

Collectors: MSB

Collection date: 22.05 .2016

31.5. Catapodium rigidum (L.) C. E., Hubbard in Dony, Fl. Bedfordshire 4371953.

Collection number: 800

Synonyms: Poa rigidaL., Cent. Apl. 1:5. 1755; Scleropoa rigida (L.) Griseb., Spicil. Fl. Rumel. 2: 431. 1846.

Distribution: W. and S. Europe, Cyprus, Syria, Palestine, Lebanon, Jordan, N. Africa, Turkey, Iran and Caucasus. - In Tarhuna: Terghlat and Zurgan. FI.April-June.

Collectors: MSB Collection date: 8.04.2016

31.6. * Cenchrus ciliaris L., Mant. Alt. 2: 302. 1771. Collection number: 851

Synonyms: Pennisetum ciliare (L.) Link, Hort. Berol. 1: 213. 1827.

Distribution: S. Europe, N. Africa, Arabia, Iran, Afghanistan, Pakistan and India. - In Tarhuna: Dawoon and Khsaya.

FI. March-April.

Collectors: MSB

Collection date:27.04.2017 
31.7. Cutandia dichotoma (Forsk.) Trabut in Batt And Trab., Fl. Alg. Mon. 237.1895.

Collection number: 812

Synonyms: Festuca dichotoma Forsk., Aeg. Arab. 22. 1775.

Distribution: Cyprus, Syria, Palestine, Jordan, Sinai, Egypt, Iran, C. Asia, N. Africa, Spain and Italy.

- In Tarhuna: Msabha.

FI. March-June.

Collectors: MSB Collection date:19.05.2016

31.8. *Cutandia maritima (L.) Barbey, FI. Sard. Comp. 72: 1885.

Collection number: 745

Synonyms: Triticum maritimumL., Sp. PI. ed. 2: 128. 1762.

Distribution: Mediterranean region. - In Tarhuna: Whedat.

FI. March.

Collectors: MSB

Collection date:22.03.2016

31.9. Cutandia memphitica (Spreng.) richter, PI. Europ. 1: 77. 1890.

Collection number: 823

Synonyms: Dactylis memphitica Spreng., Bot. Gart. Halle, Nachtr. 1: 20. 1801; Scleropoa memphitica (Spreng.) Parl., Fl. Ital. 1: 470. 1848.

Distribution: Mediterranean Europe and N. Africa, Syria, Lebanon, W. Pakistan, Afghanistan and

C. Asia. - In Tarhuna: Dawoon.

FI. February-May.

Collectors: MSB Collection date:19.05.2016

31.10. *Cyndon dactylon (L.) Pers., Syn. PI. 1: 85 (1805).

Collection number: 824

Synonyms: Panicum dactylonL., Sp. PI. 58. 1753.

Distribution: Cosmopolitan. - In Tarhuna: Khsaya and Sok-El-Ahed.

FI. April-October.

Collectors: MSB

Collection date: 19.05 .2016

31.11. *Desmazeria philistaea (Boiss.) H. Scholz, in Willdenowia, 6: 291. 1971.

Collection number: 776

Synonyms: Scleropoa philistaea Boiss., Diagn. PI. Or. Nov. Ser. I, 13: 60.1853.

Distribution: Libya, Egypt, Palestine and Syria. In Tarhuna: Hejaz.
FI. March-April.

Collectors: MSB

Collection date: 22.03 .2016

31.12. *Hordeum marinum Hudson, FI. Angl. et. 2, 1: 57.1778.

Collection number: 746

Synonyms: Hordeum maritimumwith., Nat. Arr. Br.PI. ed. 2, 1: 127. 1787.

Distribution:N. Africa, W. \& S. Europe, Syria, Palistine, Jordan, Turkey, Iran, and Afghanistan. - In Tarhuna: Dawoon, Khsaya, Sok-El-Ahed and Whedat.

FI. April-May.

Collectors: MSB

Collection date: 22.03 .2016

31.13. *Hyparrhenia hirta (L.) Stapf in Prain, FI. Trop. Afr. 9: 315. 1919.

Collection number: 834

Synonyms: Andropogon hirtusL., Sp. PI. 1046. 1753.

Distribution: Mediterranean region, S. W. Asia, Pakistan, N.W. India, S. \& E. Africa. - In Tarhuna: Msabha.

FI. March-May.

Collectors: MSB

Collection date:19.05.2016

31.14. Lagurus ovatus L., Sp. PI. 81.1753.

Collection number: 777

Distribution: Macronasia, E. France and Mediterranean region. - In Tarhuna:Hejaz.

FI. March-June.

Collectors: MSB Collection date:27.03.2016

31.15. * Lolium multiflorum Lam., Fl. France. 3: 621.1778.

Collection number: 869

Synonyms: Lolium italicum A. Braun in Flora 17: 259. 1834; L.gaudiniParl., Fl. Ital. 1: 532. 1848.

Distribution: Europe, N. Africa and temperate Asia. - In Tarhuna: Shersharah.

FI. March-May.

Collectors: MSB Collection date:27.04.2017

31.16. * Lolium rigidum Gaudin, Agrost. Helvet. 1:334 1811.

Collection number: 801

Distribution: Europe, Mediterranean region, Middle East and Caucasus. - In Tarhuna: Dawoon, Khsaya, Terghlat, Wlaad Hammad and Zurgan. FI.March-June. 
Collectors: MSB Collection date: 8.04 .2016

31.17. *Lophochloa salzmannii (Boiss.) H. Scholz in Willdenowia, 6: 292. 1971.

Collection number: 716

Synonyms: Koeleria salzmannii Boiss. et Reuter, Pugillus, 123. 1852.

Distribution: N. Africa, Iberian Peninsula. - In Tarhuna: Dawoon, Khsaya, Meghawsha, Msabha, Whedat and Zurgan.

FI. February-June. Collectors: MSB

Collection date:19.03.2016

31.18. Lygeum spartum Loefl. ex Linn., Cent. PI. ed. 5, 1: 4. 1754.

Collection number: 917

Distribution: Mediterranean region. - In Tarhuna: Hejaz.

FI.March-April.

Collectors: MSB

Collection date:12.06.2017

31.19. *Pennisetum divisum (Forsk. ex Gmel) Henr., in Blumea 3: 162. 1938.

Collection number: 889

Synonyms: Panicum divisum Forsk ex Gmel., Syst. Nat. 2: 156. 1791.

Distribution: N. Africa, Arabia, Iraq, Iran, Afghanistan, Pakistan and India. - In Tarhuna: Wlaad Hammad.

FI. March-May.

Collectors: MSB

Collection date:10.05.2017

31.20. *Stipa barbata Desf., Fl. Atlant. 1:97, tab. 27. 1798.

Collection number: 835

Distribution: Spain, Mediterranean region, Jordan, Sinai, Cacasus, Iran, Pakistan, Afghanistan, Kashmir, C. Asia and N. Africa. - In Tarhuna: Khsaya, Msabha and Terghlat.

FI.April-May.

Collectors: MSB Collection date:19.05.2016

31.21. *Stipa capensis Thunb., Prodr. FI. Cap. 19. 1794.

Collection number: 802

Synonyms: Stipa retorta Cav., Osb., S. el R. de VaIencia, 1:119. 1795; Stipa tortilis Desf., Fl. Atlant. 1: 99. T. 31. F. 1. 1798.

Distribution: Mediterranean region, N. and S. Africa, Palestine, Iraq, Iran, Afghanistan, Pakistan,
Arabia and India. - In Tarhuna: Arabeen, Khsaya, Msabha and Terghlat.

FI.February-June.

Collectors: MSB Collection date: 8.04.2016

31.22. *Stipa parviflora Desf.Fl.,Atlant. 1: 98 , tab. 29. 1798.

Collection number: 918

Distribution: Mediterranean region, Crete, Syria, Palastine, Egypt, Jordan Iran. In Tarhuna: Terghlat.

FI.March-July.

Collectors: MSB Collection date: 12.065.2017

31.23. * Stipa tenacissima L.,Cent. PI. 1: 6. 1755. Collection number: 732

Distribution: Iberian Peninsula, N. Africa, Baleares. - In Tarhuna: Dawoon, Khsaya, Mckhalef, Msabha, Terghlat, Wlaad Hammad and Zurgan.

FI.March-June.

Collectors: MSB Collection date:19.03.2016

31.24. *Stipagrostis plumosa (L.) Munro ex T. Anders. InJ. Linn. Soc. Bot. 5, Suppl. 1: 40. 1860.

Collection number: 919

Synonyms: Aristida plumoseL., Sp. PI. ed. 2. 2: 1666. 1762.

Distribution: N. Africa, Pakistan and Kashmir. - In Tarhuna: Terghlat.

FI.February-June.

Collectors: MSB

Collection date:12.06.2017

31.25. Stipagrostis pungens (Desf.) de Winter in Kirkia 3: 135. 1963.

Collection number: 903

Synonyms: Aristida pungens Desf. Fl. Atlant. 1: 109, tab. 35. 1798. Aristida pungens var. transiens Maire in M. C. 3382 bis 1941.

Distribution: W. Sahara, Egypt, Nubia, S. frica, Arabia and 'Iran. - In Tarhuna: Arabeen.

FI. May.

Collectors: MSB Collection date:10.05.2017

31.26. *Trachynia distachya (L.) Link, Hort. Bot. Berol. 1: 43. 1827.

Collection number: 803

Synonyms: Bromus distachyosL.,Cent. 2: 8. 1756, Amoen Acad. 4: 304. 1759.

Distribution: Mediterranean region and S. W. C. Asia. - In Tarhuna: Zurgan. 
FI. March-June.

Collectors: MSB

Collection date: 8.04 .2016

31.27. Vulpia ciliate Dumort, Obs. Gram. Belg. 100. 1824.

Collection number: 747

Synonyms: Festuca ciliate Danth. Ex Lam. \& DC.,Fl. Ed. 3, 3: 55. 1805.

Distribution: Mediterranean region, Turkey, Palestine, Iraq, Iran, Afghanistan, Pakistan and N.E. India. - In Tarhuna: Terghlat.

FI. March-April.

Collectors: MSB Collection date:22.03.2016

\section{Polygonaceae}

32.1. Emex spinosus (L.) Campd., Monogr, Rum. 58. 1819.

Collection number: 839

Synonyms:Rumex spinosusL., Sp. PI. 337. 1753; R. glaberForsk, Fl. Aegypt Arab. 75. 1775.

Distribution: Mediterranean costal region, Iraq, S. Iran \& Pakistan. - In Tarhuna: Sok-El-Ahed, Terghlat and Zurgan.

FI.December-June.

Collectors: MSB Collection date:19.05.2016

32.2. Polygonium equisetiforme Sibth \&Sm., Prodr, Fl. Graeca 1: 266, 1809.

Collection number: 825

Distribution: S. Europe, S.W. Asia, N. Africa, Syria, Palestine, Iraq, Iran \& Afghanistan. - In Tarhuna: Msabha, Sharshara and Terghlat.

FI.January-May.

Collectors: MSB

Collection date:19.05.2016

32.3. *Rumex bucephalophorus L., Sp. PI. 336. 1753.

Collection number: 717

Distribution: Europe, N. Africa. Syria. - In Tarhuna: Meghawsha and Zurgan.

FI.March-June.

Collectors: MSB Collection date:19.03.2016

32.4. Rumex pictus Forsk. Fl. Aeg. -Arab. 77. 1775.

Collection number: 718

Synonyms: Rumeex bipinnatus L. fil., Suppl. Plant p. 211. 1781;R. pictus ssp. bipinnatus (L.f.) Maire, Moroc. Cat. 2827. 1939.

Distribution: Morocco, Algeria, Tunisia, Libya, $\mathrm{Pa}-$ lestine and Jordan. - In Tarhuna: Meghawsha.

FI. February-May.
Collectors: MSB Collection date:19.03.2016

32.5. *Rumex tingitanus L. Sp. PI. 336. 1753.

Collection number: 878

Distribution: W. Irano-Turanian (Mauritanian Steppes), W. Mediterranean (Spain, Portugal, Italy and France). - In Tarhuna: Sok-El-Ahed.

FI. January-May.

Collectors: MSB

Collection date: 3.05 .2017

32.6. *Rumex vesicarius L., Sp. PI. 336. 1753.

Collection number: 719

Distribution: Sahao-Arabian, N. Africa, S.W. Asia

\& S. Europe. - In Tarhuna: Meghawsha.

FI. February-April.

Collectors: MSB Collection date:19.03.2016

33. Primulaceae

33.1. *Anagallis arvensis L., Sp. PI. 148. 1753.

Collection number: 748

Synonyms: Anagalis phoenicea Scopoli, FI. Carn. ed. 2. 1: 139. 1772; A.arvensis L. ssp. Phoenicea (Scop.) Vollmann in Ber. Bayer. Bot. Ges. 9: 44. 1904

Distribution: Libya, Mediterranean region, Europe, Meddle East, Iran and Pakistan. - In Tarhuna: Hejaz, Msaaed, SokOEl-Ahed, Terghlat, Whedat and Zurgan.

FI. March- may.

Collectors: MSB

Collection date:22.03.2016

33.2. Anagallis monelli L., Sp. PL. 148. 1753.

Collection number: 720

Synonyms: Anagallis linifoliaL., Sp. PI. 212. 1753. Distribution: Libya, N.W. region to MoroccO, S.W. Europe. - In Tarhuna: Meghawsha and Zurgan.

FI. March-May.

Collectors: MSB

Collection date:19.03.2016

\section{Ranunculacea}

34.1. *Adonis aestivalis L., Sp. Pl. ed. 2. 771.1762. Collection number: 920

Distribution: Europe, N. Africa - Pakistan and Central Asia. - In Targhlat.

FI. March-June.

Collectors: MSB Collection date:12.06.2017

34.2. *Adonis dentata Delile, Fl. Egypt 287, 753. f. 1813.

Collection number: 852

Synonyms: Adonis persica Boiss., Diagn. PI. Nov. 
Ser. 1. (6): 4. 1846-47.

Distribution: W. Asia, Iran, Cyprus and N. Africa. In Tarhuna: Khsaya.

FI. February-April. Collectors: MSB Collection date: 27.04.2017

34.3. Adonis microcarpa DC., Reg. Veg. Syst. Nat. 1: 223. 1817.

Collection number: 870

Synonyms: Adonis microcarpa var. intermedia Boiss., Fl. Or. 1: 18. 1869; Adonis microcarpa var. grandiflora Batt. \& Traub. Fl. Alg. 5. 1888.

Distribution: S. Europe, N. Africa, W. Asia and Iran. - In Tarhuna: Sharshara.

FI. February-April.

Collectors: MSB Collection date:27.04.2017

34.4. Delphinium halteratum Sibth. \& Smith, Fl. Graec. Prodr. 1: 37. 1809.

Collection number: 904

Synonyms: Delphinium nanum DC., Syst. 1: 349. 1818; D. peregrinum L. ssp. halteratum (Sibth. \& Smith) Batt., Fl. Alg. 16. 1888.

Distribution: Mediterranean region. - In Tarhuna: Arabeen.

Fl. December-May.

Collectors: MSB

Collection date:10.05. 2017

35. Resedaceae

35.1. Reseda lutea L., Sp. PI. 449.1753.

Collection number: 871

Synonyms: Reseda ramosissimaPour. Ex Willd., Enum. Hort. Berol. 499. 1809.

Distribution: N. Africa. - In Tarhuna: Sharshara. FI. March-May.

Collectors: MSB

Collection date:27.04.2017

36. Rhamnaceae

36.1. *Zizphus lotus (L.) Lam., Encyc. 3:317.1789.

Collection number: 826

Synonyms: Rhamnus lotus L., Sp. PI. 194. 1753.

Distribution: S. Europe, N. Africa - Arabia. - In

Tarhuna: Khsaya and Terghlat.

Fl.March-April.

Collectors: MSB

Collection date:19.05.2016

36.2. *Zizphus spina-christi (L.) Willd., Sp. PI. 1: 1105. 1798.

Collection number: 749

Synonyms: Rhamnus spina-christiL., Sp. PI. 195. 1753.

Distribution: Native of Sudan. - In Tarhuna: Whe- dat.

FI. September-April.

Collectors: MSB

Collection date:22.03.2016

37. Rubiaceae

37.1. *Galium verrucosum Huds. In Phil. Trans. Roy. Soc. B. 56: 251. 1767.

Collection number: 853

Synonyms: Galium valantia Weber in Wigg., Primit. Fl. Holsat. 12. 1780.

Distribution: S. Europe \& Mediterranean region. In Tarhuna: Sok-El-Ahed.

FI. February-May.

Collectors: MSB

Collection date: 27.04 .2017

\section{Santalaceae}

38.1. *Thesium humile Vahl. Symb. Bot. 3: 43. 1794

Collection number: 778

Distribution: Mediterranean region of Europe, N. Africa, Canaries, Egypt, S.W. Asia. - In Tarhuna: Dawoon, Hejaz and Terghlat.

FI. February-April.

Collectors: MSB Collection date:22.03.2016

\section{Scrophularaceae}

39.1. Kickxia aegyptiaca (L.) Nabelek, Iter Turc. -Pers. 3, Publ. Fac. Sci. Univ. Masaryrk 70:31. 1926.

Collection number: 733

Distribution: N. Africa to Arabia. - In Tarhuna: Dawoon, Hejaz, Mchhalef, Msaaed, Msabha, Sharshara, Sok-El-Ahed, Terghlat and Zurgan

FI. March-June.

Collectors: MSB Collection date:19.03.2016

39.2.Linaria tenuis (Viv.)Spreng.;Syst. Veg. 2:795.1825; Durand \& Baratte, FI. Lib. Prodr.179.1910; Pamp. Fl. Trip. 207. 1914; Keith. Prilim. Check List Lib.FI. 1965; N. Fein-Dotham. FI. Paleast. 3;191.1978.

Collection number: 721

Synonyms: Antirrhinum tenue Viv., Fl. Lib. 33. 1. 16. F 5-6. 1824.

Distribution: N. Africa, Palestine, Arabia.-In Tarhuna: Meghawsha.

FI. March-April.

Collectors: MSB Collection date:19.03.2016

40. Solanaceae

40.1. * Lycium chawii Roeme \& Schultes, Syst. Veg. 4: 693. 1819. 
Collection number: 921

Synonyms:Lycium persicumMiers, L. arabicum Schweinf. ex Boiss.

Distribution: N. Africa (Libya \& Egypt). S. Palestine, Arabia, Kweit, S. Iraq, Iran and Pakistan. - In Tarhuna: Terghlat.

FI.January- June.

Collectors: MSB

Collection date:12.06.2017

40.2. Lycium europaeum L., Sp. PI. 192. 1753. Collection number: 827

Synonyms: Lycium mediterraneum Dunal in DC. Pordr. 13, 1: 523. 1852.

Distribution: S. Europe, N. Africa and Asia. - In Tarhuna: Khsaya.

FI. March-April.

Collectors: MSB

Collection date:19.05.2016

40.3. Nicotina glauca R.C. Graham, Edinb. New Philos. Jour., 5: 175. 1828.

Collection number: 836

Distribution: Italy, Barse, Messine, Malta, Morocco, Algeriqa, Tunisia, Libya, Egyptr, Lebanon and Palestine. - In Tarhuna: Msabha and Terghlat.

FI. Throughout the year.

Collectors: MSB Collection date:19.05.2016

41. Zygophyllaceae

41.1. Fagonia cretica L.,Sp. PI. 386. 1753.

Collection number: 734

Distribution: A Mediterranean element; found in S. Euorpe, Canary Islands and N. Africa. - In Tarhuna: Dawoon, Hejaz, Mchhalef, Sok-El-Ahed, Terghlat, and Zurgan.

FI.October-June.

Collectors: MSB

Collection date:19.03.2016

41.2. *Peganum harmala L., Sp. PI. 444. 1753. Collection number: 779

Synonyms: Peganum dauricumPall., Reise 1: 418. 1776.

41.3. *Tribulus terrestris L., Sp. Pl. 387. 1753. Collection number:879

Synonyms: Tribulus bicornutus Fisch. \& C. A. Mey. In Bull. Soc. Nat. Mosc. 391. 1838; T. robustus Boiss. \& Noe in Boiss., Diagn. PI. Or. Nov. Ser. 2, 1: 112. 1854; Tribulus terrestris var. bicornutus (Fisch. \& C. A. Mey) Hadidi in Rech. f., Fl. Iran. 98; 17. Tab. 19. F.
Distribution: S. Europe, Asia, N. Austtalia andAfrica. - In Tarhuna: Sok-El-Ahed.

Fl. October-May.

Collectors: MSB

Collection date: 3.05 .2017

\section{RESULTS}

The flora of Tarhuna is represented by 222 different plant taxa belonging to 41 families, 143 genera, and 222 plant species of which 131 species are reported for the first time from Tarhuna. The total number of species includes three different plant groups gymnosperms with one family and one species, dicotyledons with 36 families and 185 species, and monocotyledons with 4 families and 36 species [Table 1]. The families Asteraceae, Fabaceae, Poaceae, and Lamiaceae are considered as the most sizable families with 42, 30, 27 and 11 plant species respectively [Table 2]. Onther families such as Brassicaceae, Boraginaceae, Cictaceae, and Liliaceae are represented by 10 , $8,8 \& 7$ species respectively. Whereas, the rest of the families are represented by 6 species or less. The number and percentage of the new reported plant species belonging to the largest families is also given [Table 3]. The results of this study shows that the genera such as Astragalus, Hellianthemum, and plantago are represented by 6 species each, followed by the genus Medicago with 5 species, and genera such as Convolvulus, Erodium, Euphorbia, Rumex, and stipa are represented by 4 species each. Furthermore, the rest of the genera arerepresented by $1-3$ species each. Moreover, the results have been reveled that the most characteristic features of the flora of Tarhuna is that the large number of genera [143] in proportion to that of the species [222], such character declares that each genus consists of 1-3 species. Anthemis glarosa is endemic species for Libya confined to costal belt of Libya has been collected, identified, and reportedfor the first time from Tarhuna. Considering the number of species per each family with respect to the vast area of Tarhuna the flora is not rich.

\section{DISCUSSION}

The first-time reported species from Tarhuna are representing $56.7 \%$ from 222 plant species included in this study. Such percentage gives an indication that the the flora of such region has not been considered specialy during the work on the 
Flora of Libya. Therefore, the present survey is considered as the most comperhansive study of the flora of Tarhuna. The most important characteristic features of the flora of Tarhuna is that the large number of genera in proportion to the total number of species which brings about 1-3 species per genus. One endemic plant species was also collected, identified and, reported for the first time from the region.Moreover, percentages of new reported species per each of the largest families [Table 3] exceeds 50\%, Such percentage encourages continuation of more floristic studies should be done in other regions of Libya.

\section{ACKNOWLEDGMENT}

The authors would like to express their sincere thanks to Eng. Ibrahim El- Haddar for his assistance in collecting plant specimens from Tarhuna.

Table 1. Different plant groups exist in the flora of Tarhuna.

\begin{tabular}{llll}
\hline Plant Groups & Families & Genera & Species \\
\hline Gymnosperms & 1 & 1 & 1 \\
\hline Dicotoledons & 36 & 115 & 185 \\
\hline Monocotyledones & 4 & 27 & 36 \\
\hline Total & 41 & 143 & 222 \\
\hline
\end{tabular}

Table2. The most sizable plant families present in Tarhuna.

\begin{tabular}{lll}
\hline Families & Genera & Species \\
\hline Asteraceae & 28 & 42 \\
\hline Fabaceae & 15 & 30 \\
\hline Poaceae & 19 & 27 \\
\hline Lamiaceae & 8 & 11 \\
\hline
\end{tabular}

Table 3. Number and percentage of new reported species per each of the largest familes.

\begin{tabular}{|c|c|c|c|}
\hline Families & Number of species & Number of reported species & percentage \\
\hline Asteraceae & 42 & 24 & $57.1 \%$ \\
\hline Fabaceae & 30 & 26 & $86.6 \%$ \\
\hline Poaceae & 27 & 19 & $70.3 \%$ \\
\hline Lamiaceae & 11 & 6 & $54.5 \%$ \\
\hline
\end{tabular}




\section{REFERENCES}

1. Environmental General Authority (EGA). The Fourth National Report on the Implemntation of the Convention of Biological Diversity; 2010.

2. Ascherson, P. Die aus dem mittlern Nordafrrika, dem Gebiete der Rohlfs' schen Expedition nachKufra bekannt gewordenen Pflanzen. In Rohlfs', G.: Kufra, Leipzig, 8 (1881) 386-560.

3. Boulos, L. Our present Knowledge on the Flora and Vegetation of Libya, Bibliography. Webbia 26[11] (1972) 365-400.

4. Corti, R. Flora Vegetazione del Fezzan e della Regione di Gat. Reale Soc. Geog. Italiana. (1942) 1-492.

5. Durand, E. \& Barratte, G. Florae Libycae Prodromus ou Catalogue Raisonne des Plantes de Tripolitania, Geneve, (1910) 1-330.

6. El-Gadi, A.A. Flora of Libya, 145-150. Al-Faateh Univ., Fac. Sci., Herb., Dept. Bot., Tripoli; 1988-1990.

7. Ali, S.I. and Jafri, S.M.H. Flora of Libya, 1-24, Al-Faateh Univ., Fac. Sci. Dept. Bot.,Tripoli; (1976-1977).Jafri, S. M. H. and El-Gadi, A.A. Flora of Libya, 25-144. AlFaateh Univ., Fac. Sci. Dept. Bot., Tripoli; 1977-1986.

8. Keith, H.G. A Preliminary Checklist of Libya Flora. Ministry of Agriculture Publication, Libya. 1 \& II, 1-1047 (1965) 1-528.
9. Maire, R. Flore de I'Afrque du Nord (Maroc, Algerie, Tunisia, Tripolitania, Cyrenaique et Sahara), Paris, I:229-366; II:1-374; III: 1-399; 1952-55.

10. Pampanini, R.Plantae Tripolitaniae, ab auctore anno 1913 lectae et RepertoriumFlorae vascularis Tripolitaniae, Pellas, Firenze, (1914) 1-334.

11. Pampanini, R. Prodromo della Flora Cirenaica, Ministero delle Colonie, Pub. (1931) Forli, 1-66.

12. Qaiser, M. and EL-Gadi, A. A Critical Analysis of the Flora of Libya. The Libyan Journal of Science, Volum 13 (1984).

13. Sherif, A.S. and El-Taife, A. Flora of Libya, Gymnosperms, Fac. Sci. Dept. Bot.,Al- Faateh University, Tripoli; (1986).

14. Sherif, A.S. and Ben-Othman, A. R. Checklist and Analysis of EL-Naser Forest Flora "Tripolitania", Bull. Nat. Herb. Fac. Sci. Al- Faateh University, TripoliLibya; 3 (1992).

15. Viviani, D. Florae Libcae Specimen. 1-68 (1824). 